\title{
An Extended Macro-Finance Model with Financial Factors*
}

\author{
Hans Dewachter ${ }^{\dagger}$ and Leonardo Iania ${ }^{\ddagger}$
}

\begin{abstract}
This paper extends the benchmark Macro-Finance model by introducing, next to the standard macroeconomic factors, additional liquidity-related and return forecasting factors. Liquidity factors are obtained from a decomposition of the TED spread while the return-forecasting (risk premium) factor is extracted by imposing a single factor structure on the one-period expected excess holding returns. The model is estimated on US data using MCMC techniques. Two findings stand out. First, the model outperforms significantly most structural and non-structural Macro-Finance yield curve models in terms of cross-sectional fit of the yield curve. Second, we find that financial shocks, either in the form of liquidity or risk premium shocks have a statistically and economically significant impact on the yield curve. The impact of financial shocks extends throughout the yield curve but is most pronounced at the high and intermediate frequencies.
\end{abstract}

*Please address any comments to Leonardo.Iania@econ.kuleuven.be. This paper was presented at the Magyar Nemzeti Bank (BESS), Hungary, at the Norges Bank (NB), Norway, at the 41st Annual Conference of the Money Macro and Finance Research Group (MMF), at the Young Researcher Workshop and Tutorial at the Humboldt-Universität (Berlin) and at the University of St. Gallen, Switzerland. We are grateful to Christiane Baumeister, Giulia Piccillo, Pablo Rovira Kaltwasser, Kristien Smedts, Priscilla Toffano, Michael Wickens, the seminar participants at the BESS (Budapest), at the NB (Oslo), at the MMF Conference (Bradford), at the University of St. Gallen and at the Young Researcher Workshop and Tutorial (Berlin) for useful comments. Remaining errors are the responsibility of the authors. Hans Dewachter acknowledges financial support of the Flemish Science Foundation (FWO, research project No: G.0324.08).

${ }^{\dagger}$ CES, University of Leuven, RSM Rotterdam and CESIFO

${ }^{\ddagger}$ CES, University of Leuven. 


\section{Introduction}

Macro-Finance (MF) models explain a substantial part of the yield curve dynamics in function of a limited number of macroeconomic factors. Examples of this MF approach include among others, Ang \& Piazzesi 2003, Bekaert et al. 2006, Dewachter \& Lyrio 2006, Graeve et al. 2009, Hordahl et al. 2006 and Rudebusch \& Wu 2008. Although the overall success of MF models is recognized, some issues remain. In particular, from a theoretical perspective, this class of models is restrictive in the selection of the factors impacting on the yield curve. Benchmark MF models typically only incorporate macroeconomic variables with direct impact on monetary policy and the risk-free interest rate. By focusing on these macroeconomic variables, benchmark MF models ignore potentially relevant financial factors including liquidity factors or factors accounting for shifts in risk aversion. Recent empirical studies illustrate the relevance of these financial factors. First, Cochrane \& Piazzesi 2005, Cochrane \& Piazzesi 2009) and Joslin et al. 2009 illustrate the importance of a return-generating factor for bond risk premia, not accounted for by macroeconomic factors. Second, the impact of liquidity shocks on the yield curves (swap and treasuries) has recently been documented by Longstaff et al. 2006, Feldhütter \& Lando 2008 or Christensen et al. 2009, among others.

The significant impact of liquidity and risk premium variables raises the question of the relative importance of macroeconomic and financial shocks and, consequently, of the macroeconomic information content of yield curve dynamics. In this paper, we analyze the information content of yield curve dynamics by assessing the relative importance of macroeconomic and financial shocks for the yield curve dynamics. To this end, we develop an extended Macro-Finance model (EMF), combining macroeconomic and financial factors. In particular, we extend the MF framework by augmenting the benchmark MF model with (i) a set of liquidity-related spread factors, and, (ii) a return-generating or risk premium factor. First, liquidity-related factors are introduced through a decomposition of the TED spread into a Libor spread and a T-bill spread factor (see Longstaff et al. 2006) 1 The T-bill spread (relative to the effective federal funds rate) is interpreted as a pure liquidity factor, generated by the time-varying convenience yield of owning treasuries, deriving from differential tax treatment or the preferred collateral features of the treasuries. The Libor spread (relative to effective federal funds rate) proxies for credit (counterpart-) risk premium 2 These spread factors thus link the alternative short-term interest rates and facilitate the modeling of the short end of the yield curve. Standard MF models abstract form these differences across short-term interest rates. In particular, when modeling the Libor-based yield curve, MF models assume the Libor spread to be zero while, when modeling the treasury-based yield curve, MF models assume a zero T-bill spread. These zero spread assumptions, while overall reasonable, can

\footnotetext{
${ }^{1}$ The TED spread is the difference between the three-month Libor rate and the three-month T-bill rate, the Libor spread is the spread between the three months Libor rate and the effective federal fund rate, and the T-bill is the effective federal funds rate minus the three months T-bill rate.

2 Longstaff et al. 2006 use the general collateral (GC) government repo rate as a basis to construct the spreads. We use the federal funds rate instead because it allows us to increase significantly the sample size. We also observe that, at a quarterly frequency, the effective federal funds rate is most of the time a good proxy for the repo rate. For example the correlation between the three months repo rate and the federal fund rate over the period 1991Q3 - 2008Q4 is about $99 \%$.
} 
become important model misspecifications, in particular during liquidity crises. Second, recent evidence has highlighted the fact that bond premia contain a significant predictable component, unrelated to macroeconomic factors. Cochrane \& Piazzesi 2005 and Cochrane \& Piazzesi 2009 find that a single linear combination of forward rates forecasts the one-year holding-period returns of bonds at different maturities. Duffee 2009 and Joslin et al. 2009 also find that a small number of factors has substantial explanatory power for the bond risk premia. Following this approach, we extend the benchmark MF model by allowing for a return-forecasting factor that drives the dynamics of the expected excess holding returns.

The final EMF model is an eight factor reduced-form VAR(I) model that combines macroeconomic and financial factors. In particular, the EMF model links the financial factors discussed above to the macroeconomic variables used in benchmark MF models. The macroeconomic variables consist of three observed variables - inflation, output gap and federal funds rate - and two unobserved stochastic trends, modeling the time-varying inflation target and the natural (equilibrium) real rate ${ }^{3}$ The EMF thus provides a comprehensive framework complementing and connecting to the recent financial (swap) yield curve models $\bigsqcup^{1}$ Following, among others, Ang et al. 2007, Chib \& Ergashev 2008, Graeve et al. 2009, Dewachter 2008 and Doh 2006, we use Bayesian methods to estimate and evaluate the EMF model on US data. Although computationally more demanding, the Bayesian approach has several advantages over standard full information maximum likelihood. One compelling reason for using Bayesian techniques is the fact that they allow to integrate informative priors into the estimation procedure. Appropriate priors can be useful in resolving numerical problems related to the near singularities or irregularities in the likelihood surface (see Chib \& Ergashev 2008). In particular, we use informative priors for the measurement errors by specifying a tight upper bound on the standard deviation of the measurement errors of certain macroeconomic and spread factors. This implies that five factors are 'nearly' observable, facilitating significantly the model identification and estimation. A second advantage of Bayesian analysis is that it generates a complete representation of the posterior distributions for the parameters. These distributions provide more detailed information than standard statistical analysis which is based on local approximations around the mode. The posterior densities are generated using MCMC simulation techniques. Four types of information sources are used: macroeconomic variables, yield curve variables, the TED and Eurodollar spread as well as survey data on inflation expectations 5

\footnotetext{
${ }^{3}$ The introduction of a time-varying equilibrium real rate is motivated by recent empirical evidence suggesting substantial volatility and persistence in the equilibrium real rate dynamics, e.g. Trehan \& Wu 2007 and Laubach \& Williams 2003. The high persistence of the equilibrium real rate suggests a significant impact of real rate shocks across the yield curve; i.e. the real rate may function as a second level factor (next to long-run inflation expectations).

${ }^{4}$ The latter models develop multifactor yield curve models by combining (swap) spread factors, modeling liquidity and credit risk, with standard latent level slope and curvature factors for the treasury yield curve. For instance, Longstaff et al. 2006 use a five factor affine framework, while Feldhütter \& Lando 2008 and Christensen et al. 2009 allow for a six factor state vector. The EMF model contributes to this literature by combining spread, risk premium and macroeconomic factors, which allows us to assess the relative importance of macroeconomic and financial factors to both macroeconomic and yield curve dynamics.

${ }^{5}$ Surveys of inflation expectations have been used in other, related, contexts. For instance, Kim \& Orphanides 2005 use surveys of interest rate expectations in a latent factor yield curve model.
} 
In the empirical analysis we focus on two implications of the extended model for the yield curve dynamics. First, we use the EMF model to analyze the fitting performance relative to standard MF and benchmark financial yield curve models (i.e. latent models). Although standard MF models explain a substantial part of the yield curve, they do not fit the yield curve as well as standard finance models. In particular, it is observed that most standard MF models perform significantly worse than standard finance models, e.g. a three-factor Vasicek model. This observation is especially relevant at the short end of the yield curve. The introduction of financial factors in the EMF model aims at improving the yield curve fit. Liquidity factors are in particular well-suited to improve the short end fit of the yield curve, given that they bridge the gap between the policy rate and the Treasury bill yield. Second, the extended model implies that the yield curve is determined both by macroeconomic factors and by financial factors. The EMF therefore allows evaluating the relative importance of macroeconomic factors relative to other financial factors and hence determines the value of the yield curve as an indicator of macroeconomic developments. We assess the relative importance of these two types of shocks by means of an impulse response analysis as well as a historical and a variance decomposition analysis of the yield curve dynamics.

The remainder of the paper is structured as follows. In section 2 the EMF model is introduced. We use a standard reduced form VAR containing three observed macroeconomic variables, two stochastic trends and three stationary latent factors. We provide the identification restrictions to interpret the latent factors respectively as the long-run inflation expectations, the equilibrium real rate, the spread factors and the risk premium factor. Section 3 proceeds by summarizing the econometric methodology and discusses in detail both the specification of priors and measurement equation. In section 4 we first provide a descriptive data analysis and then we focus on the empirical implications of the extended MF model along the lines discussed above, i.e. the performance of the extended model relative to the finance and the benchmark MF yield curve models and the relative importance of macroeconomic and financial factors. Section 5 performs a sub-sample and a sensitivity analysis in order to check for the robustness of our results. Finally, section 6 summarizes the main findings.

\section{Extended Macro-Finance models}

This section introduces the EMF framework. The model is built around (i) a macroeconomic part, describing in reduced form the dynamics of the macroeconomic state under the historical probability measure, and (ii) a financial part, introducing liquidity and risk premium factors. We present the state space dynamics and discuss the identification restrictions for the stochastic endpoints, the liquidity spreads and the risk premium factors. Standard arbitrage-free pricing techniques are used to derive the affine yield curve representations. 


\subsection{The Macro-Finance framework}

The EMF model is based on the standard exponentially affine modeling approach underlying much of the macro-finance literature, see Ang \& Piazzesi 2003. This approach combines a linear, discrete time, state space model with log-normal pricing kernel. Imposing no-arbitrage conditions on bond prices then results in the standard affine yield curve representation. Here, we introduce this macro-finance framework by briefly summarizing the modeling assumption concerning the state space dynamics and the pricing kernel.

The state space incorporates a state vector combining observable macroeconomic variables - inflation $\left(\pi_{t}\right)$, output gap $\left(y_{t}\right)$ and the policy interest rate $\left(i_{t}^{c b}\right)$, collected in the vector $X_{t}^{M}=\left[\pi_{t}, y_{t}, i_{t}^{c b}\right]^{\prime}$ - with a set of latent variables. Depending on their dynamics, we distinguish two types of latent variables: three stationary latent variables $l_{t}=\left[l_{1, t}, l_{2, t}, l_{3, t}\right]^{\prime}$ and two stochastic trends, $\xi_{t}=\left[\xi_{1, t}, \xi_{2, t}\right]^{\prime}$. The latent variables are collected in the vector $X_{t}^{L}=\left[l_{t}^{\prime}, \xi_{t}^{\prime}\right]^{\prime}$.

The state space dynamics are summarized by a VAR(I) model in the state vector $X_{t}=\left[X_{t}^{M \prime}, X_{t}^{L \prime}\right]^{\prime}$ :

$$
X_{t}=C+\Phi X_{t-1}+\Gamma S \varepsilon_{t}, \quad \varepsilon_{t} \sim N(0, I)
$$

with $\varepsilon_{t}=\left[\varepsilon_{t}^{M \prime}, \varepsilon_{t}^{l \prime}, \varepsilon_{t}^{\xi}\right]^{\prime}{ }^{6}$ The system matrices of the reduced-form VAR, $C, \Phi, \Gamma$ and $S$, are partitioned as follows:

$$
C=\left[\begin{array}{c}
C^{M} \\
C^{l} \\
0
\end{array}\right], \Phi=\left[\begin{array}{ccc}
\Phi^{M M} & \Phi^{M l} & \Phi^{M \xi} \\
\Phi^{l M} & \Phi^{l l} & \Phi^{l \xi} \\
0 & 0 & I
\end{array}\right], D=\Gamma S=\left[\begin{array}{ccc}
D^{M M} & 0 & D^{M \xi} \\
D^{l M} & D^{l l} & D^{M \xi} \\
0 & 0 & S^{\xi \xi}
\end{array}\right]
$$

where $D^{M M}$ and $D^{l l}$ are lower triangular and $S^{\xi \xi}$ is diagonal.

It is well known that no-arbitrage conditions, under appropriate assumptions on the stochastic discount factor (listed in Ang \& Piazzesi 2003 and Duffee 2002), generate an affine yield curve representation 7

$$
y_{t}(m)=A_{y}(m)+B_{y}(m) X_{t}
$$

where $y_{t}(m)$ denotes the time $t$ yield of a risk-free zero coupon bond with maturity $m$. The yield curve loadings, $A_{y}(m)=-a_{y}(m) / m$ and $B_{y}(m)=-b_{y}(m) / m$, are given by the no-arbitrage difference equations:

$$
\begin{aligned}
& a_{y}(m)=a_{y}(m-1)+b_{y}(m-1)\left(C-\Gamma S \Lambda_{0}\right)+\frac{1}{2} b_{y}(m-1) \Gamma S S^{\prime} \Gamma^{\prime} b_{y}(m-1)^{\prime}-\delta_{0} \\
& b_{y}(m)=b_{y}(m-1)\left(\Phi-\Gamma S \Lambda_{1}\right)-\delta_{1} .
\end{aligned}
$$

\footnotetext{
${ }^{6}$ The shocks contained in the vector $\varepsilon_{t}$ are denoted as follows: $\varepsilon_{t}=\left[\varepsilon_{\pi, t}, \varepsilon_{y, t}, \varepsilon_{i^{c b}, t}, \varepsilon_{l_{1}, t}, \varepsilon_{l_{2}, t}, \varepsilon_{l_{3}, t}, \varepsilon_{\xi_{\pi}, t}, \varepsilon_{\xi_{\rho}, t}\right]^{\prime}$.

${ }^{7}$ Implicitly, we use the following representation for the stochastic discount factor, $M_{t+1}$ :
}

$$
M_{t+1}=\exp \left(-i_{t}-\frac{1}{2} \Lambda_{t} S S^{\prime} \Lambda_{t}^{\prime}-\Lambda_{t} S \varepsilon_{t+1}\right) .
$$


Implicit in this yield curve representation is that (i) the prices of risk $\left(\Lambda_{t}\right)$ are linear in the state and that (ii) the risk-free interest rate $\left(i_{t}\right)$ can be recovered as a linear combination of the economic state:

$$
\begin{aligned}
& \Lambda_{t}=\Lambda_{0}+\Lambda_{1} X_{t}, \\
& i_{t}=\delta_{0}+\delta_{1} X_{t} .
\end{aligned}
$$

Finally, the affine specification for the prices of risk (equation (4) implies an affine representation for the risk premium. Specifically, the time $t$ expected excess holding return of a zero coupon bond with maturity $m, e h_{t}(m)$, is linearly related to the underlying economic state:

$$
e h_{t}(m)=b(m) \Gamma S \Lambda_{0}+b(m) \Gamma S \Lambda_{1} X_{t}-\frac{1}{2} b(m) \Gamma S S^{\prime} \Gamma^{\prime} b(m)^{\prime} .
$$

The interpretation of the risk premia $\left(e h_{t}(m)\right)$ is straightforward. Each (maturity-specific) risk premium is determined by the asset specific exposure to risk $(b(m) \Gamma S)$ and the market-wide prices of risk $\left(\Lambda_{0}\right.$ and $\left.\Lambda_{1} X_{t}\right)$. As such, given the exposure $b(m) \Gamma S$, risk premia are composed of a constant, $b(m) \Gamma S \Lambda_{0}$, and a time-varying state-dependent component, $b(m) \Gamma S \Lambda_{1} X_{t}$. Note that the model embeds the expectations hypothesis as a special case; i.e. by restricting $\Lambda_{1}=0$, we obtain maturity-specific but state-independent risk premia.

\subsection{Identification restrictions for latent factors}

The EMF model contains in total eight factors, five of which are latent. Without further restrictions, the latter factors do not have an unambiguous economic interpretation. In this section, we discuss identification restrictions for each of the latent factors. These restrictions impose a macroeconomic interpretation for the stochastic trends and a financial interpretation for the stationary latent factors. The two stochastic trends are respectively identified as the long-run expected inflation rate and the natural equilibrium real rate, while the three stationary latent variables are interpreted as liquidity, credit risk and risk premium factors.

\subsubsection{Stochastic endpoints for the macroeconomic state}

In line with the standard MF literature, we interpret the stochastic trends in terms of macroeconomic stochastic endpoints. These stochastic endpoints represent the (time-varying) equilibrium values for the macroeconomic variables. Specifically, following Kozicki \& Tinsley 2001 and Dewachter \& Lyrio 2006, we allow for a stochastic endpoint for inflation. Unlike standard MF models, we introduce a second stochastic endpoint accounting for time variation in the short-run equilibrium real interest rate. Using an additional stochastic endpoint for the real rate helps accommodating part of the dynamics of long-run yields and allows obtaining more realistic dynamics for long-run inflation expectations (see Dewachter \& Lyrio 2008). 
Formally, the stochastic endpoints, $\xi_{t}$, are defined in terms of the conditional long-run expectations of the observable macroeconomic variables at time $t$, as implied by the VAR(I) model:

$$
\lim _{s \rightarrow \infty} E_{t}\left[X_{t+s}^{M}\right]=T^{D} \xi_{t}
$$

where $T^{D}$ summarizes the set of cointegrating relationships between the macroeconomic variables 8 Sufficient conditions for these identification restrictions can be stated in terms of the partitioned matrices $\Phi, \Gamma S$ and $C$ :

$$
\begin{aligned}
& {\left[\begin{array}{c}
\Phi^{M \xi} \\
\Phi^{l \xi}
\end{array}\right]=(I-\tilde{\Phi})\left[\begin{array}{c}
T^{D} \\
0
\end{array}\right], \quad \tilde{\Phi}=\left[\begin{array}{cc}
\Phi^{M M} & \Phi^{M l} \\
\Phi^{l M} & \Phi^{l l}
\end{array}\right],} \\
& {\left[\begin{array}{c}
C^{M} \\
C^{l}
\end{array}\right]=(I-\tilde{\Phi})\left[\begin{array}{c}
0_{3 \times 1} \\
\bar{C}_{3 \times 1}^{l}
\end{array}\right],} \\
& {\left[\begin{array}{c}
D^{M \xi} \\
D^{l \xi}
\end{array}\right]=(I-\tilde{\Phi})\left[\begin{array}{c}
T^{D} \\
0
\end{array}\right] S^{\xi \xi},}
\end{aligned}
$$

where we, additionally, impose that all the eigenvalues of $\tilde{\Phi}$ have modulus strictly smaller than 1 .

Imposing a specific cointegration matrix $T^{D}$ yields stochastic endpoints with the required macroeconomic interpretation. In particular, the parameterization of $T^{D}$ in equation 6 implies that (i) the longrun expected inflation rate converges to the first stochastic trend, $E_{t} X_{1, t+s}^{M} \rightarrow \xi_{1, t}$, (ii) the long-run expectation for the output gap converges to zero while, (iii) the Fischer hypothesis is imposed for the short-run interest rate, implying an interpretation of the second stochastic endpoint as the equilibrium (natural) short-run real rate. Denoting the long-run expectations for inflation and the real rate by respectively $\xi_{1, t}=\pi_{t}^{*}$ and $\xi_{2, t}=\rho_{t}$, we have:

$$
\begin{aligned}
& \lim _{s \rightarrow \infty} E_{t}\left[\pi_{t+s}\right]=\xi_{1, t}=\pi_{t}^{*}, \\
& \lim _{s \rightarrow \infty} E_{t}\left[y_{t+s}\right]=0, \\
& \lim _{s \rightarrow \infty} E_{t}\left[i_{t+s}\right]=\xi_{1, t}+\xi_{2, t}=\pi_{t}^{*}+\rho_{t} .
\end{aligned}
$$

\subsubsection{Spread factors}

The liquidity and credit factors, $l_{1, t}$ and $l_{2, t}$, are identified through the time variation in the money market spread. We use a standard spread measure, i.e. the TED spread, defined as the difference between the 3-month T-bill, $i_{t}^{T}$, and the relevant unsecured money market rate, $i_{t}^{m m}$. This spread is often considered a key indicator of financial strain (market liquidity or credit risks) in money markets with increases in the

\footnotetext{
${ }^{8}$ Note that the specific parameterization of the cointegrating matrix generating the interpretation of stochastic endpoints in term of long-run inflation and real rate expectations is:

$$
T^{D}=\left[\begin{array}{ll}
1 & 0 \\
0 & 0 \\
1 & 1
\end{array}\right] .
$$
}


spread associated with increased counterparty and/or funding liquidity risk 9 Following recent studies, e.g. Longstaff et al. 2006 and Feldhütter \& Lando 2008, we model the money market (TED) spread as a function of two distinct spread factors, $l_{1, t}$ and $l_{2, t}$ :

$$
T E D_{t} \equiv i_{t}^{m m}-i_{t}^{T}=l_{1, t}+l_{2, t}
$$

Additional restrictions are required in order to identify and interpret each of the spread factors separately. To this end, we match the first spread factor, $l_{1, t}$, to the convenience yield, while the second factor, $l_{2, t}$, is related to credit risk:

$$
\begin{aligned}
& i_{t}^{T}=i_{t}^{c b}-l_{1, t}, \\
& i_{t}^{m m}=i_{t}^{c b}-l_{2, t},
\end{aligned}
$$

where $i_{t}^{c b}$ denotes the policy rate, proxying for a secure money market rate ${ }^{10}$ The T-bill spread, $l_{1, t}$, (approximately) measures the convenience yield of holding government bonds ${ }^{11}$ As documented in the literature, e.g. Longstaff et al. 2006, this spread is a measure of the flight to quality. Typically, because government bonds serve as collateral to secure loans in the money market, the relative demand for these bonds is high during liquidity crisis, leading to a lowering of government bond yields and a widening of the T-bill spread. The Libor spread, $l_{2, t}$, compares unsecured money market rates to their secure counterpart. This spread thus provides an indicator of counterparty or more general credit risks in the money market; a widening of the Libor spread typically indicates increased (perceived) credit risk exposure in money markets.

By introducing two spread factors, three types of short term interest rates are defined: the policy rate $\left(i_{t}^{c b}\right)$, the money market rate $\left(i_{t}^{m m}\right)$ and the Treasury bill rate $\left(i_{t}^{T}\right)$. From these three rates, given our focus on the treasury yield curve, we select the short-term T-bill rate as the risk-free rate. This pricing assumption is imposed (using equation $(10)$ ) by specifying $\delta_{0}$ and $\delta_{1}$ as:

$$
\delta_{0}=0, \quad \delta_{1}=[0,0,1,-1,0,0,0,0] .
$$

The dynamics of the spread factors are driven partly by exogenous financial shocks and partly by macroeconomic interactions (feedback) effects (see equation 11) The autonomous part of the spread factor dynamics consists of financial feedback effects, incorporated in $\Phi^{L L}$, and identifies financial shocks, with impact matrix $D^{L L}\left(D_{1,1}^{L L}>0\right.$ and $\left.D_{2,2}^{L L}>0\right)$. In particular, shocks to the T-bill spread and the Libor

\footnotetext{
${ }^{9}$ The TED spread is often used as a key indicator of market liquidity and credit risks. The TED spread correlates with several opinion surveys on bank lending practices published by the Senior Loan Officer, which address changes in the supply of, and demand for, bank loans to businesses and households on a quarterly basis. For example, the correlation between the TED spread and the net percentage of domestic firms reporting tightening credit standards is about 0.53 .

${ }^{10} \mathrm{~A}$ more common choice for the secure rate is $\mathrm{GC}$ secured repo rates. However, we use the central bank rate as a proxy for this rate because data for the effective federal funds rate date further back. This allows to use a larger sample in the empirical application. As highlighted in footnote2 at a quarterly frequency, the effective federal funds rate is most of the time a good proxy for the repo rate. Over the period 1991Q3 - 2008Q4, the correlation between the T-bill spread computed using the three months repo rate and the T-bill spread calculated using the the federal fund rate is above $71 \%$.

${ }^{11}$ Typically, convenience yields are caused by differential tax treatment and collateral issues of government bonds.

${ }^{12}$ Note that the macroeconomic identification conditions imposed in the previous section imply that only the transitory component of the macroeconomic variables can affect the two identified liquidity factors. This condition is necessary to obtain stationary dynamics for the two spread factors.
} 
spread are interpreted as flight to quality and credit crunch shocks, respectively. Macroeconomic dynamics also affect the spread factors through (i) possibly non-zero feedback effects from the macroeconomic state $\left(\Phi^{L M}\right)$ or (ii) contemporaneously, through the impact of macroeconomic shocks, $D^{L M}$.

\subsubsection{Return-forecasting factor}

Cochrane and Piazzesi $(2005,2009)$, Duffee 2009 and Joslin et al. 2009 argue that bond risk premia can be modeled by a limited set of factors. Cochrane \& Piazzesi 2005 extract a single return-forecasting factor from forward rates. Duffee 2009 and Joslin et al. 2009) show that risk premia can be explained by a limited set of principal components. We follow this line of research, and in particular Cochrane \& Piazzesi 2005, by imposing a single factor structure on the risk premia. To this end, we impose that the third stationary latent factor, $l_{3, t}$, drives the one-period expected excess holding returns. Through this identification restriction, this factor obtains the interpretation of a return-forecasting (-generating) factor, capturing all predictable variation in (one-period) excess returns.

Following Cochrane \& Piazzesi 2009, a single return-forecasting factor can be obtained through a set of restrictions on the time-varying component of the prices of risk, $\Lambda_{1}$. Defining the return-generating factor as the sixth element in the state vector, $l_{3, t}=X_{6, t}$, the identification restrictions are given by:

$$
\Lambda_{1,(i, j)}=0, j \neq 6 .
$$

Substituting the prices of risk restrictions in equation (5) allows expressing the (one-period) expected excess holding return on a bond with maturity $m$ as:

$$
e h_{t}(m)=b(m) \Gamma S \Lambda_{0}-\frac{1}{2} b(m) \Gamma S S^{\prime} \Gamma^{\prime} b(m)^{\prime}+\left[\sum_{i=1}^{\operatorname{dim} X} b_{1, i}(m) D_{i, j} \Lambda_{j, 6}\right] l_{3, t} .
$$

The latter equation shows that all time variation in the one-period risk premia is generated by the returngenerating factor $l_{3, t}$. In particular, one-period risk premia are a linear function of the return-forecasting factor $l_{3, t}$. Risk premia remain maturity-specific, however. The factor sensitivities $\sum_{i=1}^{\operatorname{dim} X} b_{1, i}(m) D_{i, j} \Lambda_{j, 6}$ determine the size and the sign of the maturity-specific response of the bond premia to the returngenerating factor. Unlike Cochrane \& Piazzesi 2009, who only estimate prices of risk of level shocks, we do not restrict the factor sensitivities of the bond premia, i.e. we do not restrict prices of risk $\Lambda_{i, 6}$, $i=1, \ldots, \operatorname{dim} X$. 13

The dynamics of the return-forecasting factor are modeled in analogy to the spread factors. We allow for autonomous dynamics, through a feedback effect $\left(\Phi_{3,3}^{L L}\right)$ and a risk aversion shock $\left(D_{3,3}^{L L}>0\right)$. Allowing

\footnotetext{
${ }^{13} \mathrm{~A}$ second set of conditions excludes the feedback from the return forecasting factor to the macroeconomic state. Specifically, we impose:$$
\Phi_{(i, j)}^{M, l}=0, j=3
$$

Imposing these restrictions ensures that the return forecasting factor is not present in the relevant state vector for macroeconomic dynamics as is easily verified from equation 1 .
} 
for autonomous dynamics in the return-generating factor is motivated by Cochrane \& Piazzesi 2009 and Joslin et al. 2009 who show that not all variation in risk premia is spanned by macroeconomic factors. Next to the autonomous dynamics, we allow macroeconomic factors to impact on the return-generating factor both indirectly through feedback effects $\left(\Phi^{L M}\right)$ and directly through the contemporaneous impact of macroeconomic shocks, $D^{L M} 11$

\section{Econometric methodology}

We use standard Bayesian estimation techniques to estimate the model, consisting of equations (1) and

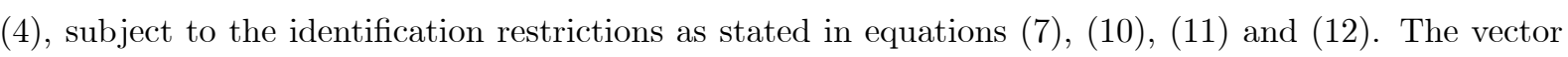
containing all parameters of the model is denoted by $\theta$. The posterior of the parameters $\theta, p\left(\theta \mid Z^{T}\right)$, is identified through Bayes rule:

$$
p\left(\theta \mid Z^{T}\right)=\frac{L\left(\theta \mid Z^{T}\right) p(\theta)}{p\left(Z^{T}\right)},
$$

with $Z^{T}$ the data set, $L()$ the likelihood function, $p(\theta)$ the priors and $p\left(Z^{T}\right)$ the marginal density of the data. The posterior density of $\theta, p\left(\theta_{i} \mid Z^{T}\right)$ is, in general, not known in closed form. We use MCMC methods, and in particular the Metropolis-Hastings algorithm, to simulate draws from the posterior. We follow the standard two-step procedure. First, a simulated annealing procedure is used to find the mode of the posterior. In a second step, the random walk Metropolis-Hastings procedure is used to trace the posterior density of $\theta \sqrt{15}$ This section describes the components of the posterior distribution, i.e. the likelihood function and the prior densities.

\subsection{Likelihood function}

The likelihood function is obtained through the prediction error decomposition implied by the transition equation (1), subject to the restrictions in equations (7), 10, 11, and 12, and the measurement equation (equation (15), discussed below. Under the assumption of normality of the structural shocks $\varepsilon_{t}$ and the measurement errors $\varepsilon_{t}^{m}$, this likelihood can be obtained using standard Kalman Filter theory (see Harvey 1991).

The measurement equation linearly relates the observation vector $Z_{t}$ to the state vector $X_{t}$ :

$$
Z_{t}=A_{m}(\theta)+B_{m}(\theta) X_{t}+\varepsilon_{t}^{m}, \varepsilon_{t}^{m} \sim N\left(0, \Sigma_{m} \Sigma_{m}^{\prime}\right)
$$

\footnotetext{
${ }^{14}$ The restrictions imposed in equation 7 imply that only transitory macroeconomic dynamics (deviations from longterm equilibrium) affect the risk premium factor. This model feature ensures the stationarity of the risk premium factor (and hence of all model-implied risk premia), which we consider an attractive feature of the model.

${ }^{15}$ The Meropolis-Hastings algorithm is based on a total of 1,000,000 simulations, with a burn-in sample of 500,000. An acceptance ratio of $25 \%$ is targeted in the algorithm. Parameters are drawn based on the Gaussian random walk model. Finally, Geweke's test for differences in means Geweke 1999) and cumulative mean plots are used to assess convergence.
} 


$$
\begin{aligned}
& A_{m}(\theta)=\left[A_{M}^{\prime}, A_{\rho}^{\prime}, A_{T b}^{\prime}, A_{y}^{\prime}, A_{s}^{\prime}, A_{c c}^{\prime}\right]^{\prime}, \quad B_{m}(\theta)=\left[B_{M}^{\prime}, B_{\rho}^{\prime}, B_{T b}^{\prime}, B_{y}^{\prime}, B_{s}^{\prime}, B_{c c}^{\prime}\right]^{\prime}, \\
& \Sigma_{m}=\operatorname{diag}\left[\Sigma_{M}, \Sigma_{\rho}, \Sigma_{T b}, \Sigma_{y}, \Sigma_{s}, \Sigma_{c c}\right]
\end{aligned}
$$

with $Z_{t}=\left[\pi_{t}, y_{t}, i_{t}^{c b}, \Delta g_{t}, y_{t}(1 / 4), y_{t}(1 / 2), \ldots, y_{t}(10), s_{t}(1), s_{t}(10), i_{t}^{\text {Libor }}-i_{t}^{c b}, i_{t}^{\text {Eurodollar }}-i_{t}^{c b}\right]$. Four types of information variables are included in the measurement equation: observable macroeconomic variables, money market information, as proxied by alternative short-term interest rates, yield curve data and survey data on inflation expectations.

Macroeconomic information. Three macroeconomic variables are incorporated in the observation vector: inflation, $\pi_{t}$, the output gap, $y_{t}$, and the policy interest rate, $i_{t}^{c b}$. Since these variables are observable, we assume zero measurement error and perfect updating. As such, the standard deviation of the measurement errors on these variables are set to zero, i.e. $\Sigma_{M}=0$. Given that these macroeconomic variables are also included in the state vector $X_{t}$, the loadings of the measurement equation are given by:

$$
A_{M}=0_{3 \times 1}, \quad B_{M}=\left[I_{3}, 0_{3 \times 5}\right]
$$

In addition, to identify the equilibrium real rate, we follow Laubach \& Williams 2003, who express the natural rate of interest as linear function of the trend growth rate of potential output, $\Delta g_{t}$ :

$$
\rho_{t}=\delta \Delta g_{t}+z_{t}
$$

where $z_{t}$ captures other determinants of $\rho_{t}$, such as households' rate of time preference. We assume $z_{t}=-c_{\rho}+\varepsilon_{z}^{m}$ and, in line with the results of Laubach \& Williams 2003, we fix $\delta$ to one. With $\rho_{t}$ being an element of the state vector, the loadings of the measurement equation related to the trend growth of potential output are identified as:

$$
A_{\rho}=c_{\rho}, B_{\rho}=[0,0,0,0,0,0,0,1]
$$

We allow for a measurement error, $\varepsilon_{\rho t}^{m}$, which captures idiosyncratic deviations of the natural rate of interest from the equilibrium value.

Yield curve information. We include yields spanning maturities between one quarter and ten years. All yields refer to government bond yields. The measurement equation loadings for the yield curve, $A_{y}$ and $B_{y}$, are obtained by imposing the no-arbitrage conditions, resulting in the affine yield curve representation (equation (2)). We allow for non-zero measurement errors for all yields (except for the 1-quarter yield), $\Sigma_{y} \geq 0$. The one-quarter T-bill is modeled using the identification conditions for the risk-free rate, i.e. equation [11, yielding $A_{T b}=\delta_{0}$ and $B_{T b}=\delta_{1}$.

Inflation expectation information. We use survey data of the expected average one- and ten-year ahead inflation rates in the observation vector, i.e. $s_{t}(1)$ and $s_{t}(10)$. Surveys of inflation expectations may provide additional information of the equilibrium inflation rate, given that these expectations are assumed to converge to the long-run inflation rate. The respective measurement equation loadings, $A_{s}$ and $B_{s}$, can 
be derived given the transition equation. In particular, solving equation (1) for the inflation expectations, we obtain the respective loadings as (see Dewachter 2008):

$$
A_{s(m)}=\frac{1}{m} e_{\pi} \sum_{j=0}^{m-1} A_{\pi}(j), B_{s(m)}=\frac{1}{m} e_{\pi} \sum_{j=0}^{m-1} B_{\pi}(j), \Sigma_{s} \geq 0
$$

and

$$
\begin{aligned}
& A_{\pi}(j)=\Phi A_{\pi}(j-1)+C, \\
& B_{\pi}(i)=\Phi B_{\pi}(i-1),
\end{aligned}
$$

with $e_{\pi}=\left[1,0_{1 \times 7}\right]$ and initial conditions $A_{\pi}(0)=0$ and $B_{\pi}(0)=I_{8}$.

Money market information. Next to the federal funds rate and the yield curve, two money market interest rates are included, i.e. the 3-month Libor and the 3-month Eurodollar rates. First, the Libor rate is used to identify the TED spread and its decomposition into the T-bill and the Libor spread. Because of limited data availability on Libor data, we also include as a second proxy the Eurodollar rate, which provides an alternative to the Libor spread and dates further back in time ${ }^{16}$ We assume (up to an idiosyncratic term) that there is a constant spread, $c_{E D}$, between Libor and Eurodollar. Each of the observed spreads, i.e. Libor and Eurodollar spread, can be thought as proxy for the theoretical Libor spread factor, modeling credit risk. The identification of the Libor spread factor 17 is thus obtained using (i) $i^{\text {Libor }}-i_{t}^{c b}=l_{2, t}+\varepsilon_{l_{2}, t}^{m}$ or (ii) $i_{t}^{\text {Eurodollar }}-i_{t}^{c b}=c_{E D}+l_{2, t}+\varepsilon_{E D, t}^{m}$, implying the following loadings for $A_{c c}$ and $B_{c c}$ :

$$
A_{c c}=\left[\begin{array}{c}
0 \\
c_{E D}
\end{array}\right], B_{c c}\left[\begin{array}{ccc}
0_{1 \times 4} & 1 & 0_{1 \times 3} \\
0_{1 \times 4} & 1 & 0_{1 \times 3}
\end{array}\right], \Sigma_{c c} \geq 0 .
$$

\subsection{Priors}

Table 1 lists the specific prior distributions used for the parameters contained in $\theta$. In general we use relatively informative priors, especially with respect to the impact matrix and the measurement errors. In this section we only discuss the most important priors.

\section{Insert Table 1}

We impose normal, relatively loose, priors on all the feedback parameters $\Phi$. The priors related to observable macroeconomic variables, $\Phi^{M M}$, are based on preliminary regression analysis. In particular, we introduce significant inertia in the macroeconomic variables (mean auto-regressive parameters between 0.5 for inflation and 0.95 for the output gap). Univariate analysis of the Libor, T-bill and TED spreads suggests that the spreads contain significant inertia. This inertia is taken into account in the prior for

\footnotetext{
${ }^{16}$ The Libor rate is an average of rates at which banks offer funds (Offer side) while the Eurodollar deposits refer to rate at which banks want to borrow funds (Bid side). Typically the Eurodollar rate is about one basis point below the Libor rate.

${ }^{17}$ Note that the T-bill spread factor is identified through the loadings $A_{T b}$ and $B_{T b}$, as discussed above.
} 
the feedback of financial factors by assuming mean autoregressive parameters in $\Phi^{L L}$ of 0.6 . Loose, zero-mean priors are used for most of the off-diagonal elements of $\Phi$. This is the case for the feedback of macroeconomic variables on liquidity and return-forecasting factors, $\Phi^{l M}$, and for the interaction across financial factors. In line with theory, a negative feedback from spread and return-forecasting factors to macroeconomic variables (i.e. $\left.\Phi^{M L}\right)$ is imposed $\mathcal{N}(-0.25,0.5)$, modeling an a priori deflationary impact of liquidity and risk-aversion shocks. Finally, we assume the standard negative feedback from the policy rate to inflation and output gap $\mathcal{N}(-0.25,0.25)$, a positive impact of output gap on inflation $\mathcal{N}(0.1,0.5)$, and, in line with the Taylor rule, a positive impact of inflation $\mathcal{N}(0.25,0.25)$ and output gap $\mathcal{N}(0.1,0.5)$ on the policy interest rate 18

The priors with respect to the impact matrix $D=\Gamma S$ in equation (1) are standard. In particular, we assume an inverted gamma distribution for the diagonal components. Depending on the type of variable, we opt for different parameterizations. An important modeling assumption in this respect is the relatively tight prior for the standard deviations of the stochastic trends, $S^{\xi \xi}, \mathcal{I} \mathcal{G}(0.002,0.2) 19$ This choice reflects the belief that the stochastic trends, in line with long-run expectations, move smoothly over time. The priors for the off-diagonal elements of $D$, contained in $D^{l M}, D^{M M}$ and $D^{l l}$, are assumed to be $\mathcal{N}(0,0.02)$. This choice leaves substantial freedom in modeling the covariance between the respective shocks.

A crucial set of uniform priors is imposed on the measurement errors. This set of priors aims at facilitating the identification of the latent factors by imposing small measurement errors for certain variables in the measurement equation. In particular, we assume zero measurement error for observable macroeconomic variables $\Sigma_{M}$ and for the three-month T-bill rate, implying $\Sigma_{T b}=0$. The latter assumption implies that the T-bill spread factor, $l_{1, t}$, becomes observable. Second, we impose an upper bound of 20 basis points on the standard deviation of the measurement errors of the Libor and Eurodollar spreads. These distributional assumptions allow for the identification of the credit-related spread factor, $l_{2, t}$, which otherwise becomes excessively volatile.

\section{Estimation results}

\subsection{Data}

The empirical analysis is performed on quarterly US data spanning the period 1960Q1 till 2008Q4. Four types of data are included in the sample: key macroeconomic series, money market indicators, yield curve data and survey data on inflation expectations. First, the macroeconomic series consist of standard measures for inflation (GDP deflator obtained from the FRED database), output gap (based on CBO

\footnotetext{
${ }^{18}$ As pointed out in note 13 the last column of $\Phi^{M, l}$ is fixed at zero in order to identify the risk premium factor.

${ }^{19}$ The first parameter of the Inverse Gamma distribution refers to the mean of the distribution while the second is the standard deviation.
} 
potential output), the policy rate (effective federal funds rate obtained from the FRED database) and the CBO-based growth of potential output. Second, we use two alternative proxies for the money market spread: the Libor spread and the Eurodollar spread. These spreads are computed relative to the effective federal funds rate and are based on the Libor and the Eurodollar three-month interest rates (source: DATASTREAM). Third, six government bond yields are included with respective maturities of 1-, 2-, 4-, 12-, 20- and 40-quarter. The yields data are compiled from the FRED database, the Gürkaynak et al. 2007 and the McCulloch-Kwon data sets 20 Finally, Surveys of Professional Forecasters data on shortand long-run average inflation expectations are used to proxy for inflation expectations.

The descriptive statistics (Table 2) of the data set are broadly in line with the stylized facts reported in the literature. In the macroeconomic dimension, we note that the average inflation (3.62\% per annum) is roughly in line with the average inflation expectations on one- and ten-year horizons, $(3.92 \%$ and $3.77 \%$ per annum), suggesting a slight average bias in inflation expectations. The CBO-based growth rate of potential output is on average $3.2 \%$ with a relatively low standard deviation of 60 basis points (p.a.). For the yield curve, our data set conforms to the standard findings reported in the literature. The yield curve is on average upward-sloping, while the volatilities of the yields are decreasing with maturity. Comparing the volatility of inflation expectations (or potential output growth) to that of the long-end of the yield curve shows a significant discrepancy between the variability in long-term yield, $2.42 \%$ p.a., and inflation expectations, $1.50 \%$ p.a., (or growth of potential output $0.6 \%$ p.a.). The long-standing belief that most variation of the long-term yields is one-to-one with long-term inflation expectations and growth of potential output is hence not recovered. Long-term yields are significantly more volatile than inflation expectations and potential output growth, generating an excess volatility puzzle. Finally, Libor and T-bill spreads are on average positive, 25 and 69 basis points, respectively. Both spreads display significant time variation, as exemplified by the standard deviations of around 50 and 108 basis points respectively.

\section{Insert Table 2}

The correlation analysis reported in Table 2 suggests strong interactions between yields and macroeconomic variables, either in the form of observable macroeconomic variables or survey data on inflation expectations. In particular, short-term yields correlate strongly with the monetary policy rate, while long-term yields correlate primarily (but not perfectly) with inflation expectations. Also, strong interactions between the financial spreads and yield curve variables are observed. Typically, the TED spread is positively correlated with the yield, indicating that money market strain is typically accompanied by high yields. Decomposing the TED spread into T-bill and Libor spreads, we observe that the correlation with the yield curve is particular pronounced and positive for the T-bill spread. Note that (except for inflation) we observe lower correlations between macroeconomic and spread factors. The strong correlations documented in Table2 suggest that a limited number of macroeconomic and financial factors drives

\footnotetext{
${ }^{20}$ The Gürkaynak et al. 2007 data set starts from the 14th of June 1961 for the 1-, 3- and 5-year bonds and from the 16th of August 1971 for the 10-year bond. The missing observations are obtained from the McCulloch-Kwon data set, available at: the http://www.econ.ohio-state.edu/jhm/ts/mckwon/mccull.htm.
} 
the yield curve.

\subsection{The performance of the EMF model}

\subsubsection{In Sample Analysis}

The overall performance of the EMF in the yield curve dimension can be assessed using the posterior distributions of the measurement errors ${ }^{21}$ In Table 3 we evaluate the model performance both in absolute and relative terms by respectively evaluating the measurement errors of the EMF model and comparing it to well-known alternative models.

Insert Table 3 and Figure 1

We first analyze the mean, the standard deviation and the autocorrelation of the measurement errors for the respective yields. Based on the statistics in Table 3 and Figure 1 we observe that the EMF model provides an excellent fit of the yield curve. On average, we obtain an R-squared of above $99 \%$ across the yield curve. Also, the means of the measurement errors are small (less than 3 basis points) and are not significantly different from zero. As implied by the high R-squared, the standard deviations of the measurement errors are small. The average standard deviation of the fitting errors is 14 basis points, ranging from a minimum of 0.01 basis points for the five-year yields to a maximum of 22.4 basis points for the ten-year yields. Despite the good fit, there is evidence of significant correlation in the measurement errors, suggesting some remaining model misspecification. This finding is common in the yield curve literature and is not specific to the EMF model 22

The bottom panels of Table 3 focus on the relative performance of the extended model. The yield curve fit of the EMF model is compared to three types of alternatives: a benchmark MF model, an $\mathrm{A}_{0}(3)$ standard affine term structure models (ATSM) yield curve model and small- and medium-scale structural MF models. The fit of the extended model is clearly superior to that of a benchmark MF model. The extended model outperforms the benchmark MF model especially in fitting the short end of the yield curve. The superiority of the EMF model clearly demonstrates the significance and economic relevance of the financial factors (both the liquidity and the risk premium factors) in modeling the yield curve. The extended model compares favorably to structural MF models, as reported in the literature. For instance, the EMF model provides a more accurate yield curve fit than the structural MF versions of Bekaert et al. 2006, Dewachter \& Lyrio 2008, Dewachter 2008 and Graeve et al. 2009). Finally, and more importantly, the extended model is competitive to benchmark finance models of the yield curve. More specifically, the EMF and the $\mathrm{A}_{0}(3)$ have comparable standard deviations (with differences of less than 6 basis points).

\footnotetext{
${ }^{21}$ The estimation results are in Table 9 to Table 11

${ }^{22}$ See Dewachter \& Lyrio 2008 for an example in the MF literature or Dai \& Singleton 2000 in the general affine class of models.
} 


\subsubsection{Out-of-sample analysis}

Given that the EMF model is an eight factor model, the risk of overfitting is present. In this subsection we show, by means of a small out-of-sample exercise, that the forecasting performance of the EMF model is comparable to that of benchmark time series models, offering a more parsimonious representations of the data. Since a full model comparison exercise is beyond the scope of this paper, we restrict the set of alternative models to the Random Walk (RW) model and a VAR (I) model on the six yields included in the data set. We estimate the models (EMF, RW and VAR(I)) starting with the sample 1960Q1-1995Q4, and produce yield forecasts up to 12 quarters ahead for each quarter of the period 1996Q1-2008Q4. Information is updated every quarter while the models are re-estimated on a yearly basis. Furthermore, when re-estimating the EMF model we exclude the parameters that were insignificant at a $20 \%$ confidence level in the estimation performed over the whole sample. This choice is not new in the literature (see, for example, Ang \& Piazzesi 2003) and, in our specific case, is directed at reducing the computational burden of the exercise 23 Table 4 reports the results of the forecasting exercise. The first three columns of the table report the root mean squared errors (RMSE) of the out-of-sample forecast, while the last two columns display the relative forecasting performance of the EMF model.

\section{Insert Table 4}

In line with many findings in the finance and macro-finance literature (e.g. Duffee 2002, Dewachter et al. 2006 and Graeve et al. 2009), it is difficult to beat the random walk model (the best model for one- and four-quarter horizons) for short forecasting horizons. For longer forecasting horizons, more than one year, the EMF outperforms both the random walk and the VAR(I) model in forecasting the short medium end of the yield curve. Furthermore, by looking at the relative performance, we notice that the EMF outperforms the VAR(I) model, except for the one-year forecast horizon. Overall, these results do not contain clear signals of overfitting.

\subsection{Factors}

The filtered time series of the eight factors, as implied by the mode of the posterior distribution, are presented in Figure 2, which also displays the 90 percent (dark shaded) and 99 percent (light shaded) error bands.

\section{Insert Figure 2}

By construction, the inflation, output gap and monetary policy factors are identified by the corresponding observable series. Focusing on the two stochastic trends, we observe that both variables are characterized by a substantial and smooth time variation. In line with Bekaert et al. 2006, Dewachter \& Lyrio 2006

\footnotetext{
${ }^{23}$ We re-optimize the model for the whole sample (i.e. we found the mode of the posterior distribution) by including only the parameters that were significant at a $20 \%$ level and the results presented in this and the following sections did not change.
} 
and Graeve et al. 2009, the long-run inflation expectations factor, $\pi_{t}^{*}$, exhibits long swings. In contrast to Bekaert et al. 2006, Dewachter \& Lyrio 2006 and Graeve et al. 2009, however, we do not observe the excess volatility of inflation expectations, typical of many benchmark models. Instead, long-run inflation expectations are aligned to survey data and come closer to typical estimates of the long-run inflation expectations as implied by pure macroeconomic models, e.g. Ireland (2007). The second stochastic trend, i.e. the natural real rate $\left(\rho_{t}\right)$, displays less inertia. Most of the variation in this factor is observed at the intermediate frequencies. The extracted real rate factor hovers between $2 \%$ and $4 \%$ p.a. and is similar to the baseline representation of the real rate in Laubach and Williams (2003). Note that, in line with the literature on the identification of the natural rate, e.g. Laubach \& Williams 2003 and Trehan $\& \mathrm{Wu} 2007$, the error bands of the real rate factor are relatively wide (almost $2 \%$ ).

Turning to the spread factors, we observe that by construction the T-bill spread is identified without error (see section 3.2 while the Libor spread factor is estimated with high precision. Both spread factors are characterized by a substantial degree of variability, fluctuating mostly within the $+/-2$ percent bounds ${ }^{24}$ The T-bill spread factor is clearly positively correlated with recession periods, indicating an increase in the spread and a flight to quality during recessions.

Finally, the return-forecasting factor displays considerable variation at all frequencies. Given the one-toone mapping between the return-forecasting factor and the risk premia, it follows that the EMF model clearly rejects the expectations hypothesis. Instead, and in line with Cochrane \& Piazzesi 2005, Duffee 2009 and Joslin et al. 2009, we find significant and persistent movements in the risk premia. Importantly, the risk premia are partially connected to the business cycle and financial crisis episodes: typically, the return-forecasting factor increases during recessions or financial crises. The return-forecasting series also shows significant low-frequency movements, broadly in line with three distinct periods for macroeconomic and financial conditions, as listed by Campbell et al. 2009. A first phase (1950s and 1960s) with stable macroeconomic conditions and low and decreasing risk aversion. The level of the return-forecasting factor for the 1960s is relatively low over this period. A second period (1970s till mid-1980s) is characterized by increasing risk aversion and high macroeconomic instability with, on average, positive and highly volatile realizations of the return-forecasting factor. A third phase (mid-1980s till 2005) in which a return to macroeconomic stability and lower risk aversion generate a more stable, trend-decreasing, return-forecasting factor.

\section{Insert Figure 3}

Figure 3 presents the factor loadings of the yield curve. These loadings represent the partial impact of the respective factors on the yield curve, assuming all else equal. Two measures of these loadings are displayed: the loadings implied by the theoretical EMF model (as implied by equations (2) and (3)), and the empirical loadings obtained from a multivariate regression of each of the yields on all the

\footnotetext{
${ }^{24}$ The large spikes observed in both the Libor and the T-bill spreads are due to the fact that we use the daily (not monthly average) of the effective federal funds rate. This rate can display peaks at given days, not followed by equivalent peaks in either the LIBOR or the T-bill rates.
} 
factors. The empirical loadings are displayed together with the $95 \%$ confidence interval. In addition, we include regression results for the yields with maturities of 8-, 16- and 36-quarter. The latter yields were not used in the estimation of the EMF model and are used as an out-of-sample gauge of the model. Several observations can be made with respect to the loadings. First, EMF-implied and empirical loadings are aligned. Most of the EMF-based loadings are within the 95\% confidence interval of the estimated coefficients, also for those yields not used in the estimation of the EMF model. The close relation between model-implied and empirical loadings, suggests that the model does not suffer from major inconsistencies. Second, the loadings indicate that different factors operate at different maturities. The short end of the yield curve is primarily sensitive to changes in the federal funds rate of the T-bill spread factor. The long end of the yield curve is affected by a variety of factors. The most important factors include the natural real interest rate, the long-run inflation expectations and the return-generating factor. Interestingly, spread factors tend to either impact primarily on the short end of the yield curve (the T-bill spread factor) or the intermediate maturities (the Libor spread), with the Libor spread acting as curvature factor.

\section{Insert Table 5}

Finally, we relate the EMF factors to those obtained in the ATSM literature. We use the first five principal components (PCs) of the yields included in the dataset as a base for the yield curve factors, where the first three PCs are interpreted as level, slope and curvature factors. Table 5 presents the estimation results (R-squared) from regressing each of the five PCs on the orthogonalized EMF factors. For example, the first column of the table reports the R-squared of regressing the first principal component on the long-run inflation expectations (first row), the two stochastic trends (second row), the two stochastic trends and the orthogonalized level of inflation (third row), and so on. Overall, the EMF model provides an interpretation of the first two PCs (level and slope) while also assigning an economic meaning to a substantial part of the third (curvature) and fourth PCs. More specifically, the regression analysis summarized in Table 5 clearly connects the level factor (1st PC) of the ATSM to the long-run inflation expectations of the EMF model. Approximately two thirds of the variation of the first principal component of the yield curve is explained by the stochastic trend for inflation, $\pi_{t}^{*}$. This is in line with the findings of Rudebusch $\& \mathrm{Wu} 2008$ and Dewachter \& Lyrio 2006, who show that the level factor is linked to the central banks' implicit inflation target as perceived by private agents. The slope factor (2nd PC) is mainly explained by orthogonalized macroeconomic factors, suggesting that the slope factor primarily captures the transitory components of the macroeconomic (i.e. business cycle) dynamics. Indeed, including the three orthogonalized macroeconomic factors increases the R-squared by more than 50 percentage points. In addition, in line with Cochrane \& Piazzesi 2009, the (orthogonalized) risk premium factor contributes significantly to the slope factor dynamics as well. Financial factors, and in particular money market spread factors, dominate the third (curvature) and fourth PC, suggesting an interpretation of these factors as liquidity or money market factors. 


\subsection{Decomposing the yield curve}

What is the relative contribution of financial and macroeconomic factors/shocks to the yield curve dynamics? To answer this question, we first identify the respective macroeconomic and financial shocks, underlying the state space dynamics. Subsequently, the contribution and relative importance of each type of shock is assessed for the yield curve factors, i.e. level, slope and curvature factors 25 In particular, we (i) use the impulse response functions (IRFs) on the state vector to identify, interpret and label the respective shocks, (ii) analyze the impact of each shock on the yield curve through the IRFs of level, slope and curvature, (iii) assess the relative importance of macroeconomic, liquidity and risk premium shocks by means of a variance decomposition and, finally, (iv) illustrate the relevance of the financial factors for yield curve dynamics by a historical decomposition.

\subsubsection{Macroeconomic and financial shocks}

The reduced form VAR(I) model identifies eight macroeconomic and financial factors and shocks. Given that factors are in general not orthogonal, we prefer to analyze the relative importance of the shocks. The identification of the type of shocks is based on an approximate interpretation of their macroeconomic and financial impact, measured by means of the IRFs. Figures 4 and 5 depict the IRFs of each of the state variables for each of the reduced-form shocks 26

\section{Insert Figures 4 and 5}

We differentiate between three types of shocks: macroeconomic, money market and risk premium shocks. Within the class of macroeconomic shocks, we distinguish five types: three transitory shocks - supply $\left(\varepsilon_{\pi}\right)$, demand $\left(\varepsilon_{y}\right)$ and monetary policy $\left(\varepsilon_{i}\right)$ shocks- and two permanent shocks - inflation target $\left(\varepsilon_{\pi^{*}}\right)$ and an equilibrium growth rate $\left(\varepsilon_{\rho}\right)$ shock. The responses of the three transitory shocks (Figure 4 panels (a) to (c)) are in line with a structural interpretation of supply, demand and policy rate shocks. Two qualifications should be kept in mind, however: the inflation response to the demand shocks is imprecisely estimated and we observe a price puzzle in the response to a policy shock. The two permanent shocks (Figure 5 panels (c) and (d)) generate the required permanent effects. An increase in the inflation target triggers a permanent increase in inflation and interest rate and generates substantial transitory expansionary effects. Increases in the equilibrium growth rate lead to transitory expansionary effects in inflation and output and a permanent increase in the interest rate (due to the higher natural real interest rate).

\footnotetext{
${ }^{25}$ The yield curve is represented in terms of the standard level, slope and curvature factors. We follow the literature in defining the level as the conditional cross-section (unweighted) average of the yield curve, the slope by the spread between the 10-year and the 3-months yield and the curvature by the difference between the 10-year minus 1-year and the 1-year minus 3 -month spread.

${ }^{26}$ In the identification of the shocks we use two measures. We consider the relative importance of a shock in the instantaneous response of the respective variables and, in addition, use the combined dynamic adjustment of the variables to each of the shocks.
} 
The IRFs identify two types of money market shocks, which we label respectively as 'flight to quality' and 'credit crunch' shocks. The flight to quality shocks primarily impact on the convenience yield (T-bill spread) while credit crunch shocks affect the money market spread (Libor spread). The flight to quality shocks typically generate a decrease in government bond yields, a decrease in inflation and a monetary policy easing. Typical credit crunch shocks, increasing the money market spread, have stagflationary effects on the economy. Somewhat controversial is the response of the policy rate, which (countering higher inflation) increases. Finally, by construction, return-generating factor shocks are neutral with respect to the macroeconomy and the money market.

\subsubsection{The yield curve: IRFs and variance decomposition}

Table6 6identifies the relative importance of the macroeconomic, money market and risk premium shocks to the variation of the level, slope and curvature factor.

\section{Insert Table 6 and Figure 7}

A first conclusion emerging from this decomposition is that financial shocks (either in the form of money market or risk premium shocks) have a significant impact throughout the yield curve and across frequencies. The important role of financial shocks in yield curve dynamics rejects the implication of standard MF models that all variation of the yield curve can be explained in terms of the standard set of macroeconomic shocks. Within the class of financial shocks, money market shocks have the most pervasive effects as they impact on each of the yield curve factors. The risk premium shocks, in contrast, predominantly affect the slope factor. Second, the relative importance of financial shocks increases with the sampling frequency. The macroeconomic information content of high-frequency changes in the yield curve is therefore limited. As indicated by Table 6, high-frequency yield curve dynamics are to a large extent dominated by either financial or monetary policy shocks. At these frequencies, we observe that a substantial part of the variation in each of the yield curve factors (level, slope and curvature) can be attributed to financial shocks. For example, at the 2-quarter forecast horizon, more than one third of the variation in the level and slope factors and more than fifty percent of the movements in the curvature factor is related to financial shocks. At the business cycle frequencies (e.g. between 8 and 40 quarters), we obtain a more pronounced effect of macroeconomic shocks on the yield curve factors. The level factor is significantly affected by supply, monetary policy and inflation target shocks. The slope factor responds to the typical business cycle factors, i.e. supply, demand and monetary policy shocks and to the returngenerating factor. The curvature factor seems to be reacting primarily to money market developments, either in the form of policy rate or flight to quality or credit crunch shocks. Finally, the EMF model, unlike standard MF models, suggests that low-frequency movements of the level factor are affected by both permanent macroeconomic shocks and financial shocks. The long-standing belief, also incorporated in standard MF models, that all (low-frequency) variation in the level can be linked to long-run inflation expectations is thus falsified. Instead, we find that only 56 percent can be attributed to shocks to the 
inflation target. Other shocks, such as equilibrium real rate shocks, monetary policy shocks and financial shocks, also account for a substantial part of the variation. Moreover, financial shocks remain important sources of variation in the slope and curvature factors also at lower frequencies.

\section{Insert Figure 6}

Figure6 6 presents the impulse response analysis for the level, slope and curvature factors. The IRFs of the level factor (Figure 6 panel (a)) indicate a transitory increase in the level of the yield curve in response to supply, demand or monetary policy shocks. A flight to quality shock (affecting the convenience yield of government bonds) decreases temporarily the level factor, while credit crunch and/or risk premium shocks induce the opposite level effect. Only shocks to the inflation target or the equilibrium real growth rate lead to permanent level effects, caused by the fact that long-run yields increase more in anticipation of future increases in the policy rate. The IRFs for the slope factor (Figure 6 panel (b)) highlight the importance of monetary policy, liquidity and risk premium shocks in the determination of the slope of the yield curve. Contractionary monetary policy shocks typically lead to a transitory decrease in the term spread. Money market shocks impact asymmetrically across the yield curve, affecting its slope. While flight to quality shocks increase the slope, credit crunch shocks have the opposite effect. Flight to quality shocks generate a strong decrease in yields, which is most pronounced for the short end of the yield curve, leading to an increase in the slope. Credit crunch shocks, in contrast, lead to a more than proportional increase in short-term yields, decreasing the slope. Furthermore, unlike money market shocks, risk premium shocks increase primarily long-term yields, resulting in an increasing slope effect. Finally, note that there is a strong link between the slope and the curvature effect: decreases (increases) in the slope are associated with a decreases (increases) in the curvature of the yield curve.

\subsubsection{Historical decomposition}

While variance decompositions and IRFs present the population values for the relative contributions, the historical decomposition of the yield curve dynamics identifies over time the relative contribution of the respective shocks to the actual realized yield curve dynamics. In this section, we illustrate the relevance of financial shocks for the yield curve dynamics by revisiting the conundrum and the recent financial crisis periods. Decomposing the yield curve dynamics over these periods clearly indicates the significance of liquidity and risk premium effects in bond markets.

\section{Insert Figures 8}

Figure 8 displays the historical decomposition of the effective federal funds rate, the 3-month T-bill rate and the 10-year yield over the period 2004 -2008. This period was first characterized by the directional divergence between short and long maturity yields ("conundrum" period, 2004-2006) and later by the financial crisis involving unprecedented cuts in the federal funds rate and sharp increases in the slope of the yield curve. The decomposition implied by Figure 8 reveals that a significant part of the increase 
in the federal funds rate over the period 2004-2006 is explained by liquidity shocks. Especially negative flight to quality shocks (e.g. search for yield shocks) caused the trend-wise increase in the federal funds rate. Macroeconomic developments (supply and policy shocks) contributed to the upward trend of the policy rate. The 3-month T-bill decomposition is similar to the federal funds rate with liquidity shocks dominating the increase in the yield. In line with the conundrum, long-maturity yields did not significantly increase over the period 2004-2006. The model attributes the disconnect between short and long rates to developments in the risk premium (return-forecasting factor), showing a significant decrease over this period. Due to the larger exposure of long-term yields to the return-forecasting factor, risk premium shocks compensated to a large extent the positive liquidity shock resulting in a stable long-run yield. A similar conclusion has been obtained by Backus \& Wright 2007), who document a significant drop in the term premia during the conundrum period. The financial crisis period (starting mid 2007) is again dominated by liquidity shocks. Both the federal funds and the 3-month T-bill rate decrease significantly in light of the sequence of flight to quality shocks. Note that credit crunch shocks as well as negative supply shocks (with deflationary effects) also contributed significantly to the fall in both interest rates. Finally, over the financial crisis period, the trend-wise decline in risk premia reverted. Positive shocks to the risk premium partially compensated for the effects of the liquidity shocks for the 10-year maturity bond. Overall, we can conclude that, over the period 2004-2008, the EMF model attributes a significant role to liquidity shocks for the money market developments.

\section{Robustness check}

We perform two types of robustness checks for the estimated EMF model. First, we analyze the robustness of the main findings over sub-samples by re-estimating the EMF model for the sample period 1986Q12008Q4. Second, we evaluate the impact of the chosen priors by comparing the results to those implied by a non-Bayesian full-information-maximum-likelihood (FIML) procedure.

\subsection{Sub-sample analysis}

Figure 9 displays the variance decomposition of the yield curve factors of an EMF model estimated over the sample period 1986Q1-2008Q4. By re-estimating over this sample, we avoid mixing different monetary policy regimes and in particular omit the Great Inflation and Disinflation periods and concentrate on a more homogeneous era in terms of macroeconomic dynamics, namely the Greenspan-Bernanke era ${ }^{27}$

\section{Insert Figure 9}

\footnotetext{
${ }^{27}$ The sub-sample analysis is normally conducted by splitting the sample in two parts and by re-estimating the model for both subsamples. In the case of our model it is difficult to apply this procedure because for two series, the ten-year inflation-expectations and the Libor rate, the data begin only in the 80's. This makes the identification of the long run inflation expectations and of the Libor spread more problematic.
} 
The results obtained in the sub-sample estimation basically mirror those obtained for the full sample. In particular, the main conclusions related to the overall importance of financial factors, i.e. money market factors and the return-forecasting factor, remain unaltered. As illustrated by Figure 9 also for the sub-sample we find evidence of (i) a significant contribution of the return-generating factor to the slope factor and (ii) money market factors (spreads) significantly contributing to each of the factors (level, slope and curvature). Minor differences are observed with respect to the importance of long-run inflation expectations, which are much more stable and hence less important in yield curve dynamics for the sub-sample.

\subsection{Sensitivity analysis}

This section shows that the main conlusions drawn in the paper do not crucially hinge on the specification of the prior (see section 3.2. To this end, we perform a sensitivity analysis by re-estimating the EMF model under the FIML assumptions.

\section{Insert Table 8}

Table 8 reports the parameters' bounds used in the optimization procedure. From the table, it can be noticed that we impose only loose constraints on the parameters vector. For example, the identification conditions related to the two liquidity factors are now removed: the T-bill spread is not observable any more $\left(\Sigma_{T b} \neq 0\right)$ ant the upper bounds for the standard deviations of the Libor and Eurodollar spreads are not constrained to be lower than 20 basis points.

\section{Insert Figure 10}

Figure 10 depicts the variance decomposition of the yield curve factors evaluated at the optimum of the likelihood function. If we look at the financial factors dimension, Figure 10 is basically identical to Figure 7 which shows the variance decomposition of the EMF evaluated at the mode of the posterior distribution. This leads us to conclude that financial shocks impact significantly the yield curve across frequencies irrespectively of the type of prior we specify for the parameters ${ }^{28}$ The only relevant differences with respect to the results reported in the previous sections are the fitting errors of the spread factors: the standard deviations of the three-month T-bill series and the Libor spread are now equal to 18 and 41 basis points, respectively. These figures are approximately 20 basis points higher than the values obtained at the mode of the posterior distribution, i.e. by incorporating the prior information. We think that the higher volatility of the two liquidity factors tends to capture outliers present in the dataset.

\footnotetext{
${ }^{28}$ The overall picture does not change also if we look at the response of the yield curve factors to shocks on the state variables: the results reported in section 4.4 are confirmed.
} 


\section{Conclusions}

In this paper we presented a MF model which incorporates financial factors and allows in addition for time variation in the long-run real rate dynamics. We estimated the extended MF model for the US over the period 1960Q1-2008Q4. Based on the estimation results, two conclusions can be drawn.

First, the extended model outperforms significantly the standard MF models in fitting the yield curve. The difference in fit is particularly pronounced for the short end of the yield curve. Allowing for liquidity and risk premium shocks, as implied by the spreads and return-forecarting factors, is crucial to this result. Interestingly, the extended MF model not only outperforms benchmark MF models, but is also comparable to standard finance models of the yield curve. This result is important as it shows that MF models offer a competitive alternative to canonical financial yield curve models.

Second, a pure macroeconomic theory of the yield curve is not in reach. The relevance of financial factors, in the form of liquidity and risk premium shocks, indicates that a significant part of the yield curve dynamics does not directly originate from macro shocks. Variance decompositions indicate that liquidity and risk premium shocks generate high- and medium-frequency changes in slope and curvature of the yield curve. Level shifts remain relatively immune to these shocks. Historical decompositions of yield curve dynamics corroborate these conclusions by pointing at the significance of liquidity and risk premium shocks.

\section{References}

Ang, A., Dong, S. \& Piazzesi, M. (2007), 'No-arbitrage taylor rules', Working Paper, National Bureau of Economic Research .

Ang, A. \& Piazzesi, M. (2003), 'A no-arbitrage vector autoregression of term structure dynamics with macroeconomic and latent variables', Journal of Monetary Economics 50(4), 745-787.

Backus, D. K. \& Wright, J. H. (2007), 'Cracking the conundrum', SSRN eLibrary .

Bekaert, G., Cho, S. \& Moreno, A. (2006), 'New-keynesian macroeconomics and the term structure', SSRN eLibrary .

Campbell, J. Y., Shiller, R. J. \& Viceira, L. M. (2009), 'Understanding inflation-indexed bond markets', Brookings Papers on Economic Activity (1), 79-120.

Chib, S. \& Ergashev, B. (2008), 'Analysis of multi-factor affine yield curve models', SSRN eLibrary .

Christensen, J. H. E., Lopez, J. A. \& Rudebusch, G. D. (2009), 'Do central bank liquidity facilities affect interbank lending rates?', Working Paper Series, Federal Reserve Bank of San Francisco . 
Cochrane, J. H. \& Piazzesi, M. (2005), 'Bond risk premia', American Economic Review 95(1), 138-160.

Cochrane, J. H. \& Piazzesi, M. (2009), 'Decomposing the yield curve', SSRN eLibrary .

Dai, Q. \& Singleton, K. J. (2000), 'Specification analysis of affine term structure models', The Journal of Finance 55(5), 1943-1978.

Dewachter, H. (2008), 'Imperfect information, macroeconomic dynamics and the yield curve : an encompassing macro-finance model', Research series, National Bank of Belgium .

Dewachter, H. \& Lyrio, M. (2006), 'Macro factors and the term structure of interest rates', Journal of Money, Credit and Banking 38(1), 119-140.

Dewachter, H. \& Lyrio, M. (2008), Learning, macroeconomic dynamics, and the term structure of interest rates, Asset prices and monetary policy, NBER.

Dewachter, H., Lyrio, M. \& Maes, K. (2006), 'A joint model for the term structure of interest rates and the macroeconomy', Journal of Applied Econometrics 21(4), 439-462.

Doh, T. (2006), 'Estimating a structural macro finance model of the term structure', Mimeo .

Duffee, G. R. (2002), 'Term premia and interest rate forecasts in affine models', The Journal of Finance $\mathbf{5 7}(1), 405-443$.

Duffee, G. R. (2009), 'Information in (and not in) the term structure', Mimeo .

Feldhütter, P. \& Lando, D. (2008), 'Decomposing swap spreads', Journal of Financial Economics 88(2), 375-405.

Geweke, J. (1999), 'Using simulation methods for bayesian econometric models: inference, development,and communication', Econometric Reviews 18(1), 1-73.

Graeve, F. D., Emiris, M. \& Wouters, R. (2009), 'A structural decomposition of the u.s. yield curve', Journal of Monetary Economics 56(4), 545 - 559 .

Gürkaynak, R. S., Sack, B. \& Wright, J. H. (2007), 'The u.s. treasury yield curve: 1961 to the present', Journal of Monetary Economics 54(8), 2291 - 2304.

Harvey, A. C. (1991), Forecasting, Structural Time Series Models and the Kalman Filter, Cambridge University Press.

Hordahl, P., Tristani, O. \& Vestin, D. (2006), 'A joint econometric model of macroeconomic and termstructure dynamics', Journal of Econometrics 131(1-2), 405-444.

Joslin, S., Priebsch, M. \& Singleton, K. J. (2009), 'Risk premium accounting in macro-dynamic term structure models', Mimeo . 
Kim, D. H. \& Orphanides, A. (2005), 'Term Structure Estimation with Survey Data on Interest Rate Forecasts', SSRN eLibrary .

Kozicki, S. \& Tinsley, P. A. (2001), 'Shifting endpoints in the term structure of interest rates', Journal of Monetary Economics 47(3), 613 - 652 .

Laubach, T. \& Williams, J. C. (2003), 'Measuring the natural rate of interest', The Review of Economics and Statistics 85(4), 1063-1070.

Longstaff, F. A., Liu, J. \& Mandell, R. E. (2006), 'The market price of credit risk: An empirical analysis of interest rate swap spreads', Journal of Business $\mathbf{7 9}(5)$, 2337-2359.

Rudebusch, G. D. \& Wu, T. (2008), 'A macro-finance model of the term structure, monetary policy and the economy', The Economic Journal 118, 906-926.

Trehan, B. \& Wu, T. (2007), 'Time-varying equilibrium real rates and monetary policy analysis', Journal of Economic Dynamics and Control 31, 1584-1609. 
Table 1: Prior Distributions of the PARAMeters

\begin{tabular}{llccc}
\hline & & Distr. & Mean & Std. Dev. \\
\hline$\Phi^{M M}(1,1)$ & & $\mathcal{N}$ & 0.500 & 0.250 \\
$\Phi^{M M}(2,1)$ & & $\mathcal{N}$ & 0.000 & 0.500 \\
$\Phi^{M M}(3,1)$ & & $\mathcal{N}$ & 0.250 & 0.250 \\
$\Phi^{M M}(1,2)$ & & $\mathcal{N}$ & 0.100 & 0.500 \\
$\Phi^{M M}(2,2)$ & & $\mathcal{N}$ & 0.950 & 0.250 \\
$\Phi^{M M}(3,2)$ & & $\mathcal{N}$ & 0.100 & 0.500 \\
$\Phi^{M M}(1,3)$ & & $\mathcal{N}$ & -0.250 & 0.250 \\
$\Phi^{M M}(2,3)$ & & $\mathcal{N}$ & -0.250 & 0.250 \\
$\Phi^{M M}(3,3)$ & & $\mathcal{N}$ & 0.800 & 0.250 \\
$\Phi^{l M}(j, i)$ & $j, i=1,2,3$ & $\mathcal{N}$ & 0.000 & 0.500 \\
$\Phi^{M l}(j, i)$ & $j=1 ; i=1,2$ & $\mathcal{N}$ & -0.250 & 0.250 \\
$\Phi^{M l}(j, i)$ & $j=2,3 ; i=1,2$ & $\mathcal{N}$ & -0.250 & 0.500 \\
$\Phi^{l l}(j, j)$ & $j=1,2,3$ & $\mathcal{N}$ & 0.600 & 0.500 \\
$\Phi^{l l}(j, i)$ & $j \neq i$ & $\mathcal{N}$ & 0.000 & 0.500 \\
$D^{M M}(j, j)$ & $j=1,2,3$ & $\mathcal{I} \mathcal{G}$ & 0.010 & 2.000 \\
$D^{M M}(j, i)$ & $j>i$ & $\mathcal{N}$ & 0.000 & 0.020 \\
\hline
\end{tabular}

\begin{tabular}{llccc}
\hline & & Distr. & Mean & Std. Dev. \\
\hline$D^{l M}(j, i)$ & $j, i=1,2,3$ & $\mathcal{N}$ & 0.000 & 0.020 \\
$D^{l l}(j, j)$ & $j=1,2,3$ & $\mathcal{I} \mathcal{G}$ & 0.010 & 2.000 \\
$D^{l l}(j, i)$ & $j>i$ & $\mathcal{N}$ & 0.000 & 0.020 \\
$S^{\xi}, \xi(j, j)$ & $j=1,2$ & $\mathcal{I} \mathcal{G}$ & 0.002 & 0.200 \\
$\Lambda_{0}(j)$ & $j=1, \ldots, 8$ & $\mathcal{N}$ & 0.000 & 20.000 \\
$\Lambda_{1}(j, 6)$ & $j=1, \ldots, 8$ & $\mathcal{N}$ & 0.000 & 100.000 \\
$\Sigma_{m}(4,4)$ & & $\mathcal{U}^{*}$ & 0.000 & 0.005 \\
$\Sigma_{m}(j, j)$ & $j=6, \ldots, 12$ & $\mathcal{U}^{*}$ & 0.000 & 0.005 \\
$\Sigma_{m}(j, j)$ & $j=13,14$ & $\mathcal{U}^{*}$ & 0.000 & 0.002 \\
$A(4)$ & & $\mathcal{N}^{\prime}$ & 0.000 & 0.010 \\
$A(14)$ & & $\mathcal{N}^{*}$ & 0.000 & 0.002 \\
$X_{0}(j)$ & $j=4,5$ & $\mathcal{U}^{*}$ & -0.015 & 0.015 \\
$X_{0}(j)$ & $j=6$ & $\mathcal{U}^{*}$ & -0.100 & 0.200 \\
$X_{0}(j)$ & $j=7,8$ & $\mathcal{U}^{*}$ & -0.010 & 0.050 \\
$\bar{C}(j)$ & $j=4,5$ & $\mathcal{U}^{*}$ & 0.000 & 0.015 \\
$\bar{C}(5)$ & & $\mathcal{U}^{*}$ & 0.000 & 0.120 \\
\hline$*$ Mean=Upper bound, Std. Dev.=Lower bound
\end{tabular}

Notes: These two panels report the priors density for the parameters estimated in the extended Macro-Finance model. $\mathcal{N}$ stands for Normal, $\mathcal{I} \mathcal{G}$ for Inverse Gamma and $\mathcal{U}$ for Uniform. The paremeters contained in the table refer to the followin state space system:

$$
\begin{aligned}
& Z_{t}=A_{m}+B_{m} X_{t}+\varepsilon_{t}^{m}, \quad \varepsilon_{t}^{m} \sim N\left(0, \Sigma_{m} \Sigma_{m}^{\prime}\right) \\
& X_{t}=C+\Phi X_{t-1}+\Gamma S \varepsilon_{t}, \quad \varepsilon_{t} \sim N(0, I)
\end{aligned}
$$

Where the observable and state vectors are

$$
\begin{aligned}
& Z_{t}=\left[\pi_{t}, y_{t}, i_{t}^{c b}, \Delta g_{t}, y_{t}(1 / 4), \ldots, y_{t}(10), s_{t}(1), s_{t}(10), i_{t}^{\text {Libor }}-i_{t}^{c b}, i_{t}^{\text {Eurodollar }}-i_{t}^{c b}\right]^{\prime} \\
& X_{t}=\left[\pi_{t}, y_{t}, i_{t}^{c b}, l_{1, t}, l_{2, t}, l_{3, t}, \xi_{1, t}, \xi_{2, t}\right]
\end{aligned}
$$

and the parameters of the state equation are given by:

$$
C=\left[\begin{array}{c}
C^{M} \\
C^{l} \\
0
\end{array}\right], \Phi=\left[\begin{array}{ccc}
\Phi^{M M} & \Phi^{M l} & \Phi^{M \xi} \\
\Phi^{l M} & \Phi^{l l} & \Phi^{l \xi} \\
0 & 0 & I
\end{array}\right], D=\Gamma S=\left[\begin{array}{ccc}
D^{M M} & 0 & D^{M \xi} \\
D^{l M} & D^{l l} & D^{M \xi} \\
0 & 0 & S^{\xi \xi}
\end{array}\right]
$$

with

$$
\left[\begin{array}{c}
C^{M} \\
C^{l}
\end{array}\right]=\left(I-\left[\begin{array}{cc}
\Phi^{M M} & \Phi^{M l} \\
\Phi^{l M} & \Phi^{l l}
\end{array}\right]\right)\left[\begin{array}{c}
0_{3 \times 1} \\
\bar{C}_{3 \times 1}^{l}
\end{array}\right]
$$

Finally the parameters $\Lambda_{0}$ and $\Lambda_{1}$ are related to the stocastic discount factor used for pricing the government bonds:

$$
M_{t+1}=\exp \left(-i_{t}-\frac{1}{2} \Lambda_{t} S S^{\prime} \Lambda_{t}^{\prime}-\Lambda_{t} S \varepsilon_{t+1}\right)
$$

with $i_{t}=y_{t}(1 / 4)$ and $\Lambda_{t}=\Lambda_{0}+\Lambda_{1} X_{t}$ 


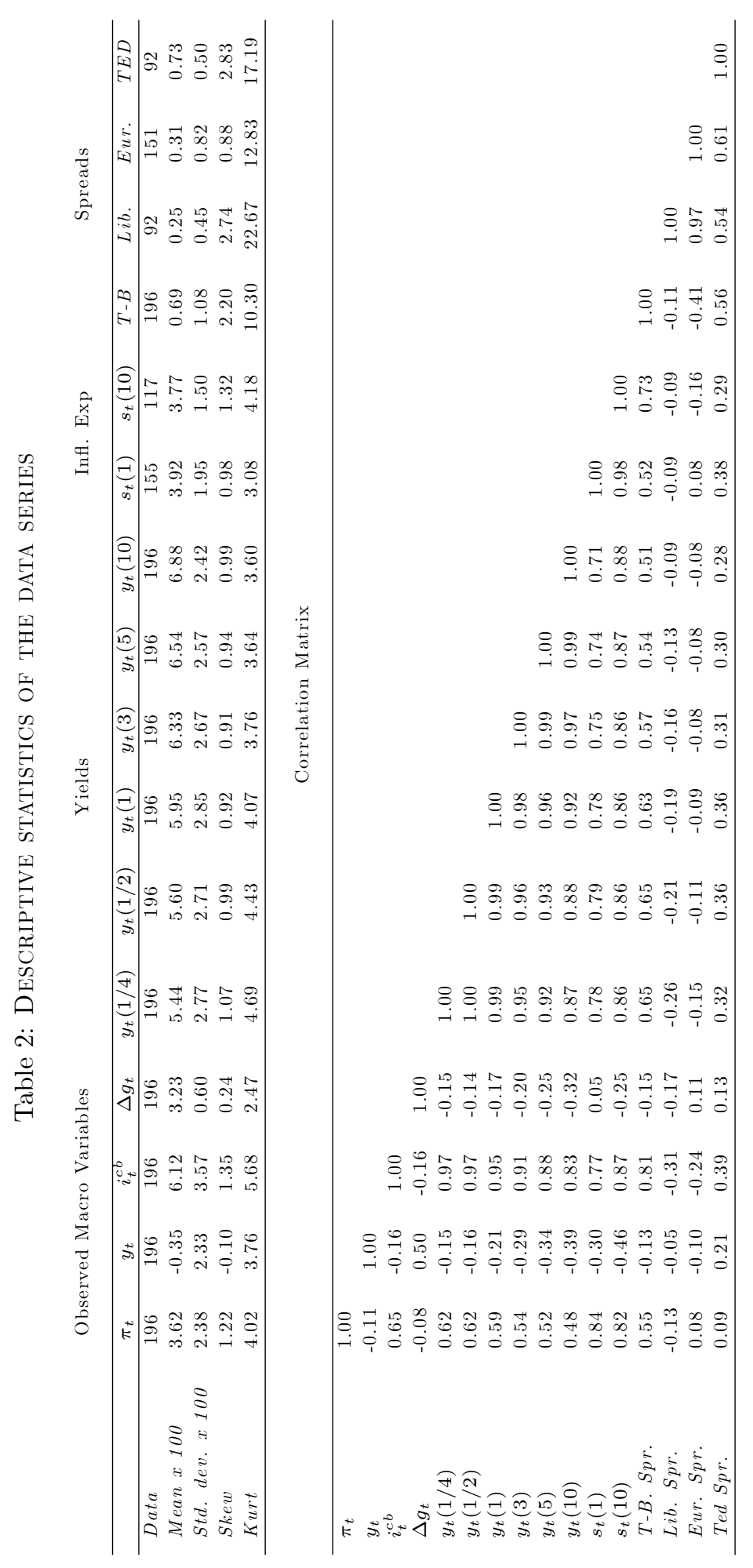

Ð

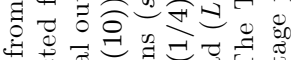

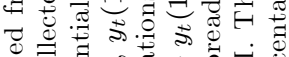

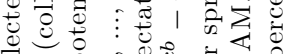

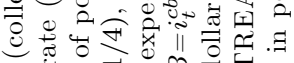

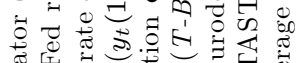

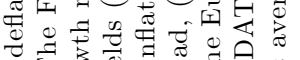

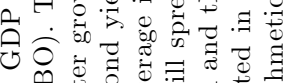

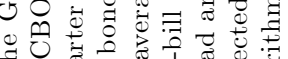
记

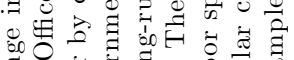

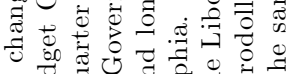

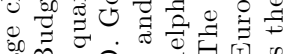

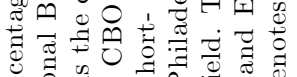
过

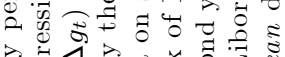

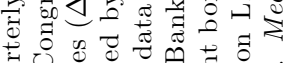
๘ ఈ

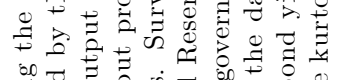
so

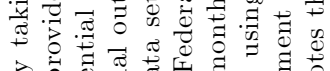
s.

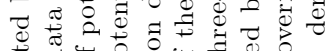

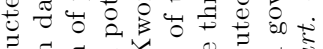

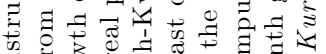

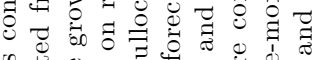
.

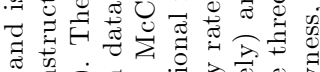

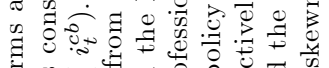

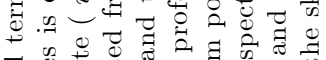
๘

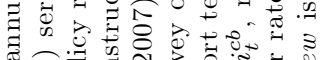

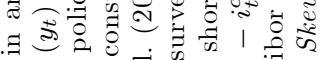
चु W

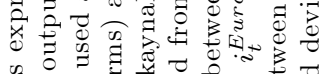

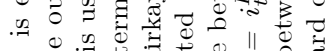

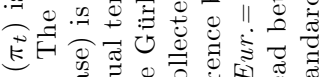

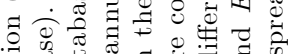

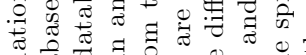

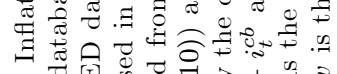

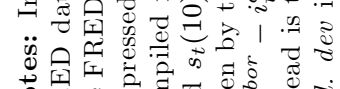

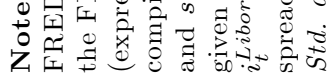


Table 3: Fit of THE YIELD CURVE

Extended Macro-Finance Model (EMF)

\begin{tabular}{llcccccc}
\hline & Yields & $1 / 4$ yr. & $1 / 2$ yr & 1 yr. & 3 yr. & 5 yr. & 10 yr. \\
\hline \multirow{3}{*}{ EMF } & Mean & $0.00^{*}$ & 0.00 & -0.01 & -0.01 & 0.00 & 0.03 \\
& Std. dev. & $0.00^{*}$ & 0.15 & 0.20 & 0.11 & 0.00 & 0.22 \\
& Auto-Corr. & $0.00^{*}$ & $0.23^{* *}$ & $0.50^{* *}$ & $0.56^{* *}$ & $0.53^{* *}$ & $0.53^{* *}$ \\
& Implied $R^{2}$ & $100.00 \%$ & $99.71 \%$ & $99.50 \%$ & $99.82 \%$ & $99.99 \%$ & $99.20 \%$ \\
\hline
\end{tabular}

Non Structural Models

\begin{tabular}{llcccccc}
\hline & Yields & $1 / 4$ yr. & 1/2 yr & 1 yr. & 3 yr. & 5 yr. & 10 yr. \\
\hline MF & Std. dev. & 1.28 & 0.47 & 0.42 & 0.19 & $0.00^{*}$ & 0.33 \\
Latent & Std. dev. & 0.14 & 0.14 & 0.16 & $0.00^{*}$ & $0.00^{*}$ & 0.16 \\
\hline
\end{tabular}

Structural Models

\begin{tabular}{llcccccc}
\hline & Yields & $1 / 4 y r$. & $1 / 2 y r$ & 1 yr. & 3 yr. & 5 yr. & $10 y r$. \\
\hline GEW(2008) & Std. dev. & - & - & 0.32 & 0.17 & $0.00 *$ & 0.28 \\
BCM(2006) & Std. dev & & & 0.45 & - & - & 0.54 \\
DL (2008) & Std. dev & - & - & 0.60 & 0.58 & 0.56 & 0.54 \\
D (2008) & Std. dev & 1.03 & 0.44 & 0.40 & 0.20 & 0.08 & 0.35 \\
\hline & $*$ set to zero &
\end{tabular}

$* *=$ significant at $5 \%$ level

Notes: The upper panel of the table shows the statistics for the fitting errors of the yield curve implied by the extended Macro-Finance model (EMF). Mean denotes the sample average per year, Std. dev is the standard deviation per year, Auto-Corr. is the first order quarterly autocorrelation and Implied $R^{2}$ denotes the implied R-squared of the EMF model. The middle panel of the table displays the estimated standard deviations of the yield measurement errors of a Macro-Finance (MF) model and of a benchmark $A_{0}(3)$ finance model, i.e. a three latent factors Vasicek model. The bottom panel of the table shows estimated standard deviations of the yield measurement errors of four structural Macro-Finance models. For these structural models the following abbreviations are used: GEW(2008) is the model of Graeve et al. 2009, BCM(2006) is the model of Bekaert et al. 2006, DL (2008) is the model of Dewachter \& Lyrio 2008 and D (2008) is the model of Dewachter 2008. 
Table 4: OUt-OF -AMPLE FORECAST

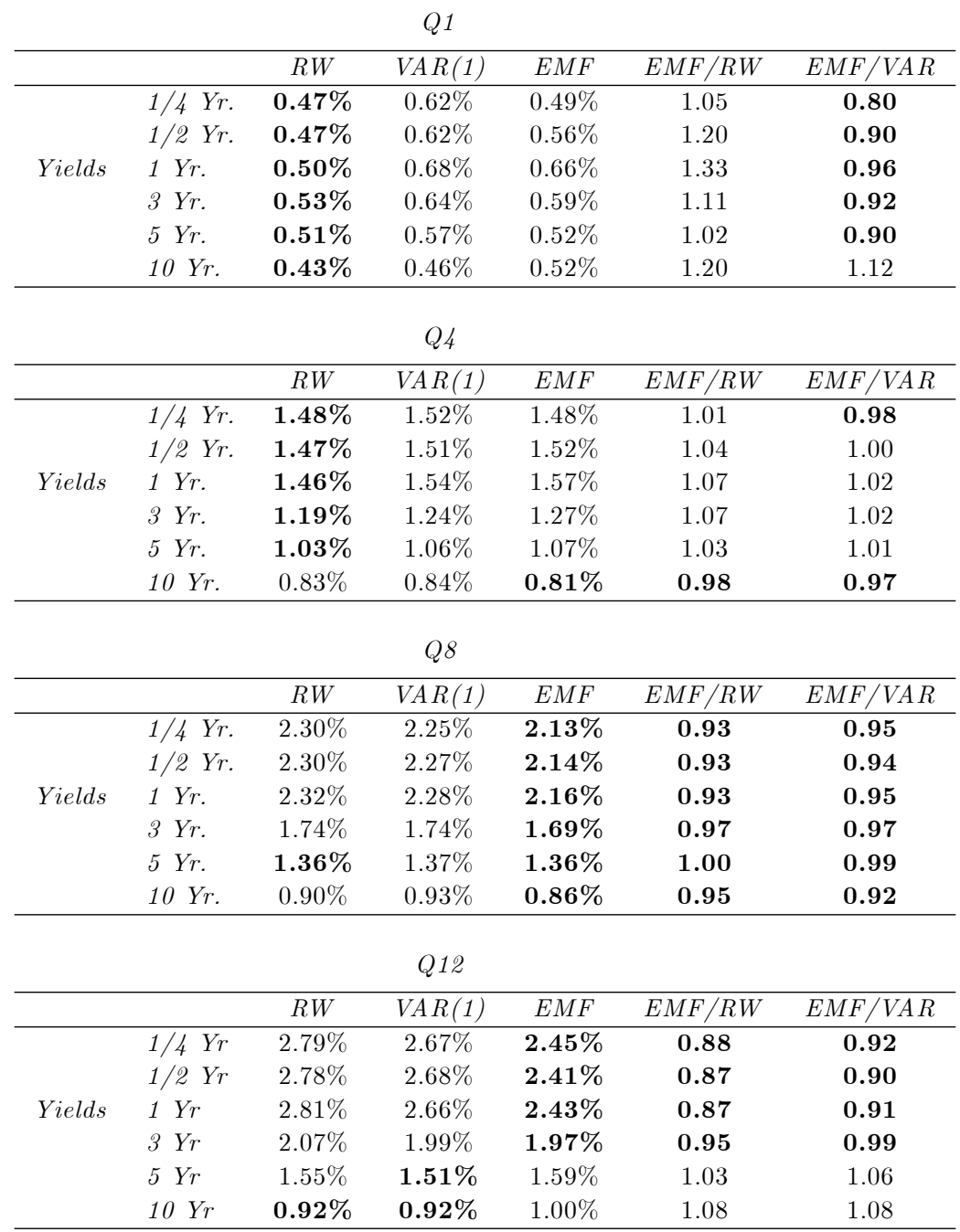

Notes: The table, column 2 to 4 , presents the annualized percentage root means squared errors (RMSE) for four models: the random walk model (RW), the VAR(I) and the Extended Macro-Finance model (EMF). The last two columns present the ratio of RMSE of EMF model with respect to the other two models. The forecasts are obtained (i) by estimating the models over the period 1960Q1-1995Q4 and (ii) by producing yields forecasts up to 12 quarters ahead for each quarter of the period 1996Q1-2008Q4. Information is updated every quarter while the models are re-estimated on a yearly basis. For the EMF model, we present the RMSE for a model re-estimated including only the parameters that were significant at the $20 \%$ confidence level (in the estimation conducted over the whole sample). 
Table 5: Fraction of Yields' Principal COMponents explained By the EXtended MacroFINANCE MODEL

\begin{tabular}{lccccc} 
& \multicolumn{5}{c}{ Principal Component } \\
\hline (Orthogonalized) factors & $1^{\text {st }}$ & $2^{\text {nd }}$ & $3^{\text {rd }}$ & $4^{\text {th }}$ & $5^{\text {th }}$ \\
\hline$\pi_{t}^{*}$ & $66.19 \%$ & $4.15 \%$ & $7.08 \%$ & $3.07 \%$ & $0.02 \%$ \\
$\pi_{t}^{*}, \rho_{t}$ & $73.67 \%$ & $9.93 \%$ & $12.58 \%$ & $8.33 \%$ & $8.49 \%$ \\
$\pi_{t}^{*}, \rho_{t}, \epsilon_{\pi_{t}}$ & $73.86 \%$ & $31.29 \%$ & $12.75 \%$ & $9.51 \%$ & $12.44 \%$ \\
$\pi_{t}^{*}, \rho_{t}, \epsilon_{\pi_{t}}, \epsilon_{y_{t}}$ & $74.36 \%$ & $43.85 \%$ & $14.54 \%$ & $10.91 \%$ & $14.78 \%$ \\
$\pi_{t}^{*}, \rho_{t}, \epsilon_{\pi_{t}}, \epsilon_{y_{t}}, \epsilon_{i_{t}^{c b}}$ & $94.30 \%$ & $64.49 \%$ & $15.37 \%$ & $11.68 \%$ & $17.53 \%$ \\
$\pi_{t}^{*}, \rho_{t}, \epsilon_{\pi_{t}}, \epsilon_{y_{t}}, \epsilon_{i_{t}^{c b}}, \epsilon_{i_{t}^{c b}-i_{t}^{T}}$ & $97.71 \%$ & $66.38 \%$ & $16.73 \%$ & $16.95 \%$ & $17.58 \%$ \\
$\pi_{t}^{*}, \rho_{t}, \epsilon_{\pi_{t}}, \epsilon_{y_{t}}, \epsilon_{i_{t}^{c b}}^{c b}, \epsilon_{i_{t}^{c b}-i_{t}^{T}}, \epsilon_{i_{t}^{m m}}-i_{t}^{c b}$ & $97.93 \%$ & $66.39 \%$ & $43.53 \%$ & $39.06 \%$ & $17.95 \%$ \\
$\pi_{t}^{*}, \rho_{t}, \epsilon_{\pi_{t}}, \epsilon_{y_{t}}, \epsilon_{i_{t}^{c b}}, \epsilon_{i_{t}^{c b}-i_{t}^{T}}, \epsilon_{i_{t}^{m m}}-i_{t}^{c b}, \epsilon_{l_{3, t}}$ & $99.95 \%$ & $98.49 \%$ & $57.74 \%$ & $41.15 \%$ & $18.97 \%$ \\
\hline
\end{tabular}

Notes: This table reports the estimation results (R-squared) from regressing each of the first five principal components (PC) of the one-, two-, four-, twelve-, twenty- and forty-quarter yields on the (orthogonalized) factors of the EMF model (evaluated at the mode of the posterior distribution of the parameters). For example, the first column of the table reports the R-squared of regressing the first PC on the long run inflation expectations (first row), the two stochastic trends (second row), the two stochastic trends and the orthogonalized level of inflation (third row), and so on. We othogonalize all factors except the two stochastic trends, $\pi_{t}^{*}$ and $\rho_{t}$. The orthogonal component of each factor is obtained by taking the residuals of the regression of the factor of interest on the the two stochastic trends and the previously orthogonalized factors. For example, we obtain the orthogonal component of the output gap by taking the residuals series of the regression of $y_{t}$ on the long run inflation expectations $\left(\pi_{t}^{*}\right)$, the equilibrium real rate $\left(\rho_{t}\right)$ and the orthogonal component of the inflation series $\left(\epsilon_{\pi_{t}}\right)$ 
Table 6: Variance Decomposition of the Yields Curve factors

Level Factor

\begin{tabular}{lcccccccc}
\hline Shocks & Supply & Demand & Mon. pol. & Folight to qual. & Credit cr. & Risk premia & Infl. target & Eq. gr. rate \\
\hline $1 Q$ & $0.45 \%$ & $2.49 \%$ & $53.44 \%$ & $29.21 \%$ & $2.27 \%$ & $10.19 \%$ & $1.52 \%$ & $0.42 \%$ \\
$2 Q$ & $0.81 \%$ & $2.61 \%$ & $49.46 \%$ & $29.18 \%$ & $4.67 \%$ & $10.69 \%$ & $2.01 \%$ & $0.56 \%$ \\
$4 Q$ & $1.76 \%$ & $2.80 \%$ & $44.18 \%$ & $28.36 \%$ & $7.36 \%$ & $11.47 \%$ & $3.18 \%$ & $0.89 \%$ \\
$10 Q$ & $3.40 \%$ & $3.03 \%$ & $36.06 \%$ & $25.82 \%$ & $8.50 \%$ & $12.67 \%$ & $8.37 \%$ & $2.15 \%$ \\
$40 Q$ & $2.32 \%$ & $2.02 \%$ & $21.93 \%$ & $16.24 \%$ & $5.37 \%$ & $9.27 \%$ & $35.49 \%$ & $7.36 \%$ \\
$100 Q$ & $1.34 \%$ & $1.16 \%$ & $12.62 \%$ & $9.34 \%$ & $3.09 \%$ & $5.34 \%$ & $55.98 \%$ & $11.14 \%$ \\
\hline
\end{tabular}

Slope Factor

\begin{tabular}{lcccccccc}
\hline Shocks & Supply & Demand & Mon. pol. & Flight to qual. & Credit cr. & Risk premia & Infl. target & Eq. gr. rate \\
\hline $1 Q$ & $1.96 \%$ & $4.98 \%$ & $49.54 \%$ & $13.66 \%$ & $0.50 \%$ & $25.66 \%$ & $3.01 \%$ & $0.69 \%$ \\
$2 Q$ & $2.72 \%$ & $5.40 \%$ & $44.36 \%$ & $13.76 \%$ & $2.50 \%$ & $27.47 \%$ & $3.11 \%$ & $0.68 \%$ \\
$4 Q$ & $5.04 \%$ & $5.92 \%$ & $36.69 \%$ & $12.44 \%$ & $7.23 \%$ & $28.90 \%$ & $3.15 \%$ & $0.63 \%$ \\
$10 Q$ & $10.60 \%$ & $7.05 \%$ & $27.06 \%$ & $10.06 \%$ & $11.03 \%$ & $30.57 \%$ & $3.09 \%$ & $0.54 \%$ \\
$40 Q$ & $11.98 \%$ & $8.00 \%$ & $24.03 \%$ & $9.04 \%$ & $10.94 \%$ & $32.63 \%$ & $2.90 \%$ & $0.49 \%$ \\
$100 Q$ & $11.97 \%$ & $7.99 \%$ & $24.03 \%$ & $9.04 \%$ & $10.94 \%$ & $32.63 \%$ & $2.90 \%$ & $0.49 \%$ \\
\hline
\end{tabular}

Curvature Factor

\begin{tabular}{lcccccccc}
\hline Shocks & Supply & Demand & Mon. pol. & Flight to qual. & Credit cr. & Risk premia & Infl. target & Eq. gr. rate \\
\hline $1 Q$ & $3.40 \%$ & $4.44 \%$ & $31.16 \%$ & $18.84 \%$ & $38.67 \%$ & $0.45 \%$ & $2.68 \%$ & $0.37 \%$ \\
$2 Q$ & $5.70 \%$ & $5.05 \%$ & $31.62 \%$ & $18.86 \%$ & $34.98 \%$ & $0.48 \%$ & $2.92 \%$ & $0.37 \%$ \\
$4 Q$ & $9.45 \%$ & $6.01 \%$ & $30.14 \%$ & $18.95 \%$ & $31.40 \%$ & $0.53 \%$ & $3.14 \%$ & $0.37 \%$ \\
$10 Q$ & $14.30 \%$ & $7.68 \%$ & $26.16 \%$ & $18.76 \%$ & $28.88 \%$ & $0.63 \%$ & $3.25 \%$ \\
$40 Q$ & $15.06 \%$ & $8.67 \%$ & $25.21 \%$ & $18.49 \%$ & $28.30 \%$ & $0.73 \%$ & $3.21 \%$ \\
$100 Q$ & $15.08 \%$ & $8.66 \%$ & $25.22 \%$ & $18.48 \%$ & $28.29 \%$ & $0.73 \%$ & $3.21 \%$ \\
\hline
\end{tabular}

Notes: This table reports the forecasting error variance decomposition (computed at the mode of the posterior distribution of the parameters) of the yield curve factors, i.e. the level, the slope and the curvature factor. The level factor is the conditional cross-section (unweighted) average of the yield curve. The slope is the spread between the 10 -year and the 3-month yield. The curvature is the difference between the 10-year minus 1-year and the 1-year minus 3-month spread. Mon. pol. stands for Monetary policy, Flight to qual. for Flight to quality, Credit cr. for Credit crunch, Infl. target for Inflation target and Eq. gr. rate for Equilibrium growth rate. 
Table 7: Variance Decomposition State Variables

Inflation

\begin{tabular}{|c|c|c|c|c|c|c|c|c|}
\hline Shocks & Supply & Demand & Mon. pol. & Flight to qual. & Credit $\mathrm{cr}$. & Risk premia & Infl. target & Eq. gr. rate \\
\hline $1 Q$ & $99.90 \%$ & $0.00 \%$ & $0.00 \%$ & $0.00 \%$ & $0.00 \%$ & $0.00 \%$ & $0.10 \%$ & $0.00 \%$ \\
\hline $2 Q$ & $98.42 \%$ & $0.00 \%$ & $0.00 \%$ & $0.39 \%$ & $0.93 \%$ & $0.00 \%$ & $0.25 \%$ & $0.00 \%$ \\
\hline $4 Q$ & $95.12 \%$ & $0.00 \%$ & $0.05 \%$ & $1.12 \%$ & $2.92 \%$ & $0.00 \%$ & $0.77 \%$ & $0.01 \%$ \\
\hline $10 Q$ & $88.05 \%$ & $0.02 \%$ & $1.02 \%$ & $2.13 \%$ & $5.29 \%$ & $0.00 \%$ & $3.46 \%$ & $0.03 \%$ \\
\hline $40 Q$ & $68.13 \%$ & $0.02 \%$ & $1.76 \%$ & $2.23 \%$ & $5.03 \%$ & $0.00 \%$ & $22.79 \%$ & $0.04 \%$ \\
\hline $100 Q$ & $46.27 \%$ & $0.01 \%$ & $1.20 \%$ & $1.52 \%$ & $3.42 \%$ & $0.00 \%$ & $47.56 \%$ & $0.02 \%$ \\
\hline
\end{tabular}

\begin{tabular}{|c|c|c|c|c|c|c|c|c|}
\hline Shocks & Supply & Demand & Mon. pol. & Flight to qual. & Credit cr. & Risk premia & Infl. target & Eq. gr. rate \\
\hline $1 Q$ & $1.56 \%$ & $98.39 \%$ & $0.00 \%$ & $0.00 \%$ & $0.00 \%$ & $0.00 \%$ & $0.04 \%$ & $0.00 \%$ \\
\hline $2 Q$ & $2.46 \%$ & $93.99 \%$ & $2.78 \%$ & $0.54 \%$ & $0.07 \%$ & $0.00 \%$ & $0.15 \%$ & $0.02 \%$ \\
\hline $4 Q$ & $5.07 \%$ & $82.04 \%$ & $11.12 \%$ & $0.52 \%$ & $0.63 \%$ & $0.00 \%$ & $0.56 \%$ & $0.06 \%$ \\
\hline $10 Q$ & $13.84 \%$ & $50.91 \%$ & $26.30 \%$ & $1.71 \%$ & $5.12 \%$ & $0.00 \%$ & $1.93 \%$ & $0.19 \%$ \\
\hline $40 Q$ & $23.05 \%$ & $28.23 \%$ & $29.05 \%$ & $5.60 \%$ & $10.87 \%$ & $0.00 \%$ & $2.95 \%$ & $0.25 \%$ \\
\hline $100 Q$ & $23.10 \%$ & $28.15 \%$ & $29.02 \%$ & $5.62 \%$ & $10.90 \%$ & $0.00 \%$ & $2.95 \%$ & $0.25 \%$ \\
\hline
\end{tabular}

\begin{tabular}{lcccccccc}
\multicolumn{7}{c}{ Fed rate } \\
\hline Shocks & Supply & Demand & Mon. pol. & Flight to qual. & Credit cr. & Risk premia & Infl. target & Eq. gr. rate \\
\hline $1 Q$ & $2.49 \%$ & $0.67 \%$ & $96.83 \%$ & $0.00 \%$ & $0.00 \%$ & $0.00 \%$ & $0.02 \%$ & $0.00 \%$ \\
$2 Q$ & $3.73 \%$ & $1.54 \%$ & $89.50 \%$ & $3.39 \%$ & $1.82 \%$ & $0.00 \%$ & $0.01 \%$ & $0.00 \%$ \\
$4 Q$ & $6.12 \%$ & $2.81 \%$ & $75.67 \%$ & $9.06 \%$ & $6.29 \%$ & $0.00 \%$ & $0.02 \%$ & $0.03 \%$ \\
$10 Q$ & $10.63 \%$ & $4.43 \%$ & $60.24 \%$ & $12.86 \%$ & $10.89 \%$ & $0.00 \%$ & $0.67 \%$ \\
$40 Q$ & $10.08 \%$ & $4.49 \%$ & $48.95 \%$ & $11.27 \%$ & $9.76 \%$ & $0.00 \%$ & $12.76 \%$ & $2.70 \%$ \\
$100 Q$ & $7.67 \%$ & $3.41 \%$ & $37.20 \%$ & $8.57 \%$ & $7.42 \%$ & $0.00 \%$ & $29.79 \%$ & $5.94 \%$ \\
\hline
\end{tabular}

T-bill spread

\begin{tabular}{lcccccccc}
\hline Shocks & Supply & Demand & Mon. pol. & Flight to qual. & Credit cr. & Risk premia & Infl. target & Eq. gr. rate \\
\hline $1 Q$ & $3.10 \%$ & $0.93 \%$ & $53.07 \%$ & $42.47 \%$ & $0.00 \%$ & $0.00 \%$ & $0.40 \%$ & $0.04 \%$ \\
$2 Q$ & $5.37 \%$ & $0.75 \%$ & $58.36 \%$ & $34.54 \%$ & $0.10 \%$ & $0.00 \%$ & $0.80 \%$ & $0.08 \%$ \\
$4 Q$ & $8.58 \%$ & $1.25 \%$ & $57.92 \%$ & $29.22 \%$ & $1.55 \%$ & $0.00 \%$ & $1.37 \%$ \\
$10 Q$ & $12.23 \%$ & $2.42 \%$ & $51.30 \%$ & $27.15 \%$ & $4.93 \%$ & $0.00 \%$ & $1.83 \%$ & $0.13 \%$ \\
$40 Q$ & $13.01 \%$ & $2.95 \%$ & $49.72 \%$ & $26.80 \%$ & $5.49 \%$ & $0.00 \%$ & $1.87 \%$ \\
$100 Q$ & $13.02 \%$ & $2.95 \%$ & $49.72 \%$ & $26.80 \%$ & $5.49 \%$ & $0.00 \%$ & $1.87 \%$ \\
\hline
\end{tabular}

Libor spread

\begin{tabular}{lcccccccc}
\hline Shocks & Supply & Demand & Mon. pol. & Flight to qual. & Credit cr. & Risk premia & Infl. target & Eq. gr. rate \\
\hline $1 Q$ & $0.02 \%$ & $0.39 \%$ & $22.22 \%$ & $15.91 \%$ & $61.46 \%$ & $0.00 \%$ & $0.00 \%$ & $0.00 \%$ \\
$2 Q$ & $0.34 \%$ & $1.24 \%$ & $22.35 \%$ & $14.13 \%$ & $61.93 \%$ & $0.00 \%$ & $0.00 \%$ & $0.00 \%$ \\
$4 Q$ & $1.48 \%$ & $2.93 \%$ & $21.68 \%$ & $13.40 \%$ & $60.50 \%$ & $0.00 \%$ & $0.01 \%$ \\
$10 Q$ & $3.57 \%$ & $4.99 \%$ & $21.25 \%$ & $12.69 \%$ & $57.35 \%$ & $0.00 \%$ & $0.14 \%$ & $0.00 \%$ \\
$40 Q$ & $6.18 \%$ & $5.06 \%$ & $22.08 \%$ & $12.33 \%$ & $53.86 \%$ & $0.00 \%$ & $0.46 \%$ \\
$100 Q$ & $6.20 \%$ & $5.06 \%$ & $22.07 \%$ & $12.34 \%$ & $53.83 \%$ & $0.00 \%$ & $0.04 \%$ \\
\hline
\end{tabular}

Return-forecasting factor

\begin{tabular}{lcccccccc}
\hline Shocks & Supply & Demand & Mon. pol. & Flight to qual. & Credit cr. & Risk premia & Infl. target & Eq. gr. rate \\
\hline $1 Q$ & $6.54 \%$ & $0.35 \%$ & $18.42 \%$ & $8.33 \%$ & $0.07 \%$ & $66.29 \%$ & $0.00 \%$ & $0.00 \%$ \\
$2 Q$ & $5.83 \%$ & $0.33 \%$ & $17.12 \%$ & $8.93 \%$ & $0.06 \%$ & $67.72 \%$ & $0.00 \%$ & $0.00 \%$ \\
$4 Q$ & $4.96 \%$ & $0.34 \%$ & $15.30 \%$ & $9.40 \%$ & $0.05 \%$ & $69.94 \%$ & $0.00 \%$ & $0.00 \%$ \\
$10 Q$ & $4.07 \%$ & $0.46 \%$ & $12.81 \%$ & $9.24 \%$ & $0.04 \%$ & $73.38 \%$ & $0.00 \%$ & $0.00 \%$ \\
$40 Q$ & $3.84 \%$ & $0.74 \%$ & $11.65 \%$ & $8.74 \%$ & $0.06 \%$ & $74.94 \%$ & $0.00 \%$ & $0.01 \%$ \\
$100 Q$ & $3.84 \%$ & $0.75 \%$ & $11.66 \%$ & $8.74 \%$ & $0.07 \%$ & $74.93 \%$ & $0.00 \%$ \\
\hline
\end{tabular}

Notes: This table reports the forecasting error variance decomposition of the state variables, computed at the mode of the posterior distribution of the parameters. Mon. pol. stands for Monetary policy, Flight to qual. for Flight to quality, Credit cr. for Credit crunch, Infl. target for Inflation target and Eq. gr. rate for Equilibrium growth rate. 
Table 8: Bounds of The PARAMeters in The MAXimization of THE Likelihood FunCtion

\begin{tabular}{llcc}
\hline & & Max & Min. \\
\hline$\Phi^{M M}(j, i)$ & $j \neq i$ & 3.750 & -3.750 \\
$\Phi^{M M}(j, j)$ & $j=1,2,3$ & 3.750 & 0.000 \\
$\Phi^{M l}(j, i)$ & $j=1,2,3 ; i=1,2$ & 3.750 & -3.750 \\
$\Phi^{l M}(j, i)$ & $j, i=1,2,3$ & 3.750 & -3.750 \\
$\Phi^{l l}(j, j)$ & $j=1,2$ & 3.750 & 0.000 \\
$\Phi^{l l}(j, i)$ & $j \neq i$ & 3.750 & -3.750 \\
$D^{M M}(j, j)$ & $j=1,2,3$ & 0.050 & 0.000 \\
$D^{M M}(j, i)$ & $j>i$ & 0.100 & -0.100 \\
$D^{l M}(j, i)$ & $j, i=1,2,3$ & 0.100 & -0.100 \\
$D^{l l}(j, j)$ & $j=1,2,3$ & 0.050 & 0.000 \\
$S^{\xi, \xi}(j, j)$ & $j=1,2$ & 0.050 & 0.000 \\
\hline
\end{tabular}

\begin{tabular}{llcc}
\hline & & Max & Min. \\
\hline$\Lambda_{0}(j)$ & $j=1, \ldots, 8$ & 1000 & -1000 \\
$\Lambda_{1}(j, 6)$ & $j=1, \ldots, 8$ & 1000 & -1000 \\
$\Sigma_{m}(j, j)$ & $j=4, \ldots, 12$ & 0.050 & 0.000 \\
$A(4)$ & & 0.050 & -0.050 \\
$A(14)$ & & 0.015 & -0.015 \\
$X_{0}(j)$ & $j=4,5$ & 0.015 & -0.015 \\
$X_{0}(6)$ & & 0.500 & -0.100 \\
$X_{0}(j)$ & $j=7,8$ & 0.050 & -0.010 \\
$\bar{C}(4)$ & & 0.015 & 0.000 \\
$\bar{C}(5)$ & & 0.120 & 0.000 \\
$\bar{C}(6)$ & & 0.300 & 0.000 \\
\hline
\end{tabular}

Notes: These two panels report the bounds imposed to the parameters space when we maximize the likelihood function in Eq. 14. The paremeters contained in the table refer to the followin state space system:

$$
\begin{array}{lll}
Z_{t} & =A_{m}+B_{m} X_{t}+\varepsilon_{t}^{m}, \varepsilon_{t}^{m} \sim N\left(0, \Sigma_{m} \Sigma_{m}^{\prime}\right) & \text { (Meas. Eq.) } \\
X_{t} & =C+\Phi X_{t-1}+\Gamma S \varepsilon_{t}, \quad \varepsilon_{t} \sim N(0, I) & \text { (Trans. Eq.) }
\end{array}
$$

Where the observable and state vectors are

$$
\begin{aligned}
& Z_{t}=\left[\pi_{t}, y_{t}, i_{t}^{c b}, \Delta g_{t}, y_{t}(1 / 4), \ldots, y_{t}(10), s_{t}(1), s_{t}(10), i_{t}^{L i b o r}-i_{t}^{c b}, i_{t}^{\text {Eurodollar }}-i_{t}^{c b}\right]^{\prime} \\
& X_{t}=\left[\pi_{t}, y_{t}, i_{t}^{c b}, l_{1, t}, l_{2, t}, l_{3, t}, \xi_{1, t}, \xi_{2, t}\right]
\end{aligned}
$$

and the parameters of the state equation are given by:

$$
C=\left[\begin{array}{c}
C^{M} \\
C^{l} \\
0
\end{array}\right], \Phi=\left[\begin{array}{ccc}
\Phi^{M M} & \Phi^{M l} & \Phi^{M \xi} \\
\Phi^{l M} & \Phi^{l l} & \Phi^{l \xi} \\
0 & 0 & I
\end{array}\right], D=\Gamma S=\left[\begin{array}{ccc}
D^{M M} & 0 & D^{M \xi} \\
D^{l M} & D^{l l} & D^{M \xi} \\
0 & 0 & S^{\xi \xi}
\end{array}\right]
$$

with

$$
\left[\begin{array}{c}
C^{M} \\
C^{l}
\end{array}\right]=\left(I-\left[\begin{array}{cc}
\Phi^{M M} & \Phi^{M l} \\
\Phi^{l M} & \Phi^{l l}
\end{array}\right]\right)\left[\begin{array}{c}
0_{3 \times 1} \\
\bar{C}_{3 \times 1}^{l}
\end{array}\right]
$$

Finally the parameters $\Lambda_{0}$ and $\Lambda_{1}$ are related to the stocastic discount factor used for pricing the government bonds:

$$
M_{t+1}=\exp \left(-i_{t}-\frac{1}{2} \Lambda_{t} S S^{\prime} \Lambda_{t}^{\prime}-\Lambda_{t} S \varepsilon_{t+1}\right)
$$

with $i_{t}=y_{t}(1 / 4)$ and $\Lambda_{t}=\Lambda_{0}+\Lambda_{1} X_{t}$ 
Table 9: Prior and Posterior Distribution of the Phi Matrix

\begin{tabular}{|c|c|c|c|c|c|c|c|c|c|c|}
\hline & \multicolumn{3}{|c|}{ Prior } & \multicolumn{7}{|c|}{ Posterior } \\
\hline & Distr. & Mean & Std.Dev. & $0.5 \%$ & $5 \%$ & $50 \%$ & $95 \%$ & $99.5 \%$ & Mode & Mean \\
\hline$\Phi^{M M}(1,1)$ & $\mathcal{N}$ & 0.500 & 0.250 & 0.657 & 0.689 & 0.740 & 0.783 & 0.801 & 0.758 & 0.739 \\
\hline$\Phi^{M M}(2,1)$ & $\mathcal{N}$ & 0.000 & 0.500 & -0.138 & -0.088 & -0.025 & 0.034 & 0.067 & -0.009 & -0.026 \\
\hline$\Phi^{M M}(3,1)$ & $\mathcal{N}$ & 0.250 & 0.250 & 0.035 & 0.065 & 0.120 & 0.191 & 0.218 & 0.104 & 0.127 \\
\hline$\Phi^{M M}(1,2)$ & $\mathcal{N}$ & 0.100 & 0.500 & -0.028 & -0.017 & 0.005 & 0.028 & 0.040 & 0.002 & 0.005 \\
\hline$\Phi^{M M}(2,2)$ & $\mathcal{N}$ & 0.950 & 0.250 & 0.857 & 0.879 & 0.922 & 0.955 & 0.972 & 0.922 & 0.921 \\
\hline$\Phi^{M M}(3,2)$ & $\mathcal{N}$ & 0.100 & 0.500 & 0.046 & 0.061 & 0.092 & 0.131 & 0.159 & 0.094 & 0.094 \\
\hline$\Phi^{M M}(1,3)$ & $\mathcal{N}$ & -0.250 & 0.250 & 0.009 & 0.030 & 0.067 & 0.105 & 0.128 & 0.069 & 0.067 \\
\hline$\Phi^{M M}(2,3)$ & $\mathcal{N}$ & -0.250 & 0.250 & -0.173 & -0.139 & -0.076 & -0.016 & 0.019 & -0.062 & -0.078 \\
\hline$\Phi^{M M}(3,3)$ & $\mathcal{N}$ & 0.800 & 0.250 & 0.880 & 0.929 & 1.014 & 1.083 & 1.137 & 0.989 & 1.017 \\
\hline$\Phi^{l M}(1,1)$ & $\mathcal{N}$ & 0.000 & 0.500 & 0.000 & 0.033 & 0.086 & 0.140 & 0.161 & 0.075 & 0.092 \\
\hline$\Phi^{l M}(2,1)$ & $\mathcal{N}$ & 0.000 & 0.500 & -0.035 & -0.010 & 0.025 & 0.069 & 0.089 & 0.029 & 0.022 \\
\hline$\Phi^{l M}(3,1)$ & $\mathcal{N}$ & 0.000 & 0.500 & -0.047 & 0.004 & 0.145 & 0.252 & 0.313 & 0.123 & 0.138 \\
\hline$\Phi^{l M}(1,2)$ & $\mathcal{N}$ & 0.000 & 0.500 & -0.028 & -0.005 & 0.029 & 0.071 & 0.085 & 0.025 & 0.031 \\
\hline$\Phi^{l M}(2,2)$ & $\mathcal{N}$ & 0.000 & 0.500 & -0.084 & -0.073 & -0.053 & -0.036 & -0.027 & -0.049 & -0.053 \\
\hline$\Phi^{l M}(3,2)$ & $\mathcal{N}$ & 0.000 & 0.500 & -0.093 & -0.050 & -0.004 & 0.040 & 0.063 & 0.015 & -0.007 \\
\hline$\Phi^{l M}(1,3)$ & $\mathcal{N}$ & 0.000 & 0.500 & 0.071 & 0.110 & 0.192 & 0.254 & 0.294 & 0.177 & 0.192 \\
\hline$\Phi^{l M}(2,3)$ & $\mathcal{N}$ & 0.000 & 0.500 & -0.117 & -0.090 & -0.044 & 0.001 & 0.023 & -0.043 & -0.044 \\
\hline$\Phi^{l M}(3,3)$ & $\mathcal{N}$ & 0.000 & 0.500 & -0.286 & -0.204 & -0.048 & 0.109 & 0.180 & -0.057 & -0.047 \\
\hline$\Phi^{M l}(1,1)$ & $\mathcal{N}$ & -0.250 & 0.250 & -0.227 & -0.152 & -0.024 & 0.103 & 0.176 & -0.038 & -0.021 \\
\hline$\Phi^{M l}(2,1)$ & $\mathcal{N}$ & -0.250 & 0.500 & -0.370 & -0.273 & -0.101 & 0.076 & 0.184 & -0.169 & -0.094 \\
\hline$\Phi^{M l}(3,1)$ & $\mathcal{N}$ & -0.250 & 0.500 & -0.708 & -0.586 & -0.433 & -0.209 & -0.152 & -0.403 & -0.428 \\
\hline$\Phi^{M l}(1,2)$ & $\mathcal{N}$ & -0.250 & 0.250 & 0.065 & 0.158 & 0.288 & 0.431 & 0.484 & 0.279 & 0.296 \\
\hline$\Phi^{M l}(2,2)$ & $\mathcal{N}$ & -0.250 & 0.500 & -0.283 & -0.187 & -0.013 & 0.161 & 0.252 & -0.053 & -0.012 \\
\hline$\Phi^{M l}(3,2)$ & $\mathcal{N}$ & -0.250 & 0.500 & 0.187 & 0.355 & 0.509 & 0.680 & 0.804 & 0.496 & 0.516 \\
\hline$\Phi^{l l}(1,1)$ & $\mathcal{N}$ & 0.600 & 0.500 & -0.020 & 0.076 & 0.227 & 0.446 & 0.528 & 0.254 & 0.192 \\
\hline$\Phi^{l l}(2,1)$ & $\mathcal{N}$ & 0.000 & 0.500 & -0.084 & -0.027 & 0.087 & 0.191 & 0.242 & 0.104 & 0.235 \\
\hline$\Phi^{l l}(3,1)$ & $\mathcal{N}$ & 0.000 & 0.500 & -0.931 & -0.656 & -0.130 & 0.305 & 0.516 & -0.093 & 0.086 \\
\hline$\Phi^{l l}(1,2)$ & $\mathcal{N}$ & 0.000 & 0.500 & -0.212 & -0.074 & 0.071 & 0.236 & 0.334 & 0.058 & 0.077 \\
\hline$\Phi^{l l}(2,2)$ & $\mathcal{N}$ & 0.600 & 0.500 & 0.232 & 0.303 & 0.411 & 0.506 & 0.586 & 0.435 & 0.407 \\
\hline$\Phi^{l l}(3,2)$ & $\mathcal{N}$ & 0.000 & 0.500 & -0.567 & -0.397 & -0.089 & 0.257 & 0.482 & -0.017 & -0.120 \\
\hline$\Phi^{l l}(3,3)$ & $\mathcal{N}$ & 0.600 & 0.500 & 0.845 & 0.875 & 0.915 & 0.956 & 0.981 & 0.918 & 0.913 \\
\hline
\end{tabular}

Notes: This table reports the priors and the posterior density for the parameters of $\Phi$ matrix in Eq. 1 The first three columns report the distributions, means and standard deviations of the prior distributions. The fourth to the eight columns report the .5 -th, 5 -th, the 50 -th and the 95 -th 99.5 -th percentile of the posterior distributions, respectively. The last two columns report the modes and the means of the posterior distributions. All results were obtained using the Metropolis Hastings algorithm. 
Table 10: Prior and Posterior Distribution of the Impact Matrix

\begin{tabular}{|c|c|c|c|c|c|c|c|c|c|c|}
\hline & \multicolumn{3}{|c|}{ Prior } & \multicolumn{7}{|c|}{ Posterior } \\
\hline & Distr. & Mean & Std. Dev. & $0.5 \%$ & $5 \%$ & $50 \%$ & $95 \%$ & $99.5 \%$ & Mode & Mean \\
\hline$D^{M M}(1,1)$ & $\mathcal{I G}$ & 0.010 & 2.000 & 1.005 & 1.050 & 1.142 & 1.241 & 1.305 & 1.140 & 1.143 \\
\hline$D^{M M}(2,1)$ & $\mathcal{N}$ & 0.000 & 0.020 & -0.242 & -0.187 & -0.092 & 0.003 & 0.056 & -0.092 & -0.092 \\
\hline$D^{M M}(3,1)$ & $\mathcal{N}$ & 0.000 & 0.020 & -0.005 & 0.079 & 0.239 & 0.416 & 0.553 & 0.227 & 0.263 \\
\hline$D^{M M}(2,2)$ & $\mathcal{I G}$ & 0.010 & 2.000 & 0.657 & 0.686 & 0.745 & 0.815 & 0.860 & 0.729 & 0.748 \\
\hline$D^{M M}(3,2)$ & $\mathcal{N}$ & 0.000 & 0.020 & -0.192 & -0.050 & 0.095 & 0.258 & 0.344 & 0.117 & 0.092 \\
\hline$D^{M M}(3,3)$ & $\mathcal{I G}$ & 0.010 & 2.000 & 1.273 & 1.328 & 1.447 & 1.578 & 1.644 & 1.414 & 1.443 \\
\hline$D^{l M}(1,1)$ & $\mathcal{N}$ & 0.000 & 0.020 & -0.018 & 0.050 & 0.154 & 0.252 & 0.314 & 0.143 & 0.160 \\
\hline$D^{l M}(2,1)$ & $\mathcal{N}$ & 0.000 & 0.020 & -0.143 & -0.092 & -0.007 & 0.075 & 0.128 & -0.008 & -0.007 \\
\hline$D^{l M}(3,1)$ & $\mathcal{N}$ & 0.000 & 0.020 & -2.334 & -2.075 & -0.998 & -0.286 & 0.030 & -0.817 & -1.097 \\
\hline$D^{l M}(1,2)$ & $\mathcal{N}$ & 0.000 & 0.020 & -0.228 & -0.168 & -0.082 & 0.011 & 0.083 & -0.078 & -0.083 \\
\hline$D^{l M}(2,2)$ & $\mathcal{N}$ & 0.000 & 0.020 & -0.176 & -0.129 & -0.042 & 0.035 & 0.081 & -0.040 & -0.046 \\
\hline$D^{l M}(3,2)$ & $\mathcal{N}$ & 0.000 & 0.020 & -0.651 & -0.515 & -0.234 & 0.108 & 0.444 & -0.188 & -0.201 \\
\hline$D^{l M}(1,3)$ & $\mathcal{N}$ & 0.000 & 0.020 & 0.481 & 0.526 & 0.608 & 0.704 & 0.756 & 0.590 & 0.608 \\
\hline$D^{l M}(2,3)$ & $\mathcal{N}$ & 0.000 & 0.020 & -0.432 & -0.384 & -0.300 & -0.223 & -0.180 & -0.301 & -0.302 \\
\hline$D^{l M}(3,3)$ & $\mathcal{N}$ & 0.000 & 0.020 & 0.248 & 0.646 & 1.355 & 2.080 & 2.493 & 1.371 & 1.433 \\
\hline$D^{l l}(1,1)$ & $\mathcal{I} \mathcal{G}$ & 0.010 & 2.000 & 0.471 & 0.497 & 0.541 & 0.593 & 0.621 & 0.528 & 0.543 \\
\hline$D^{l l}(2,1)$ & $\mathcal{N}$ & 0.000 & 0.020 & -0.380 & -0.336 & -0.260 & -0.188 & -0.150 & -0.254 & -0.261 \\
\hline$D^{l l}(3,1)$ & $\mathcal{N}$ & 0.000 & 0.020 & -1.975 & -1.602 & -0.896 & -0.188 & 0.466 & -0.922 & -0.916 \\
\hline$D^{l l}(2,2)$ & $\mathcal{I} \mathcal{G}$ & 0.010 & 2.000 & 0.444 & 0.471 & 0.521 & 0.579 & 0.614 & 0.500 & 0.522 \\
\hline$D^{l l}(3,2)$ & $\mathcal{N}$ & 0.000 & 0.020 & -1.141 & -0.663 & 0.033 & 0.599 & 1.394 & 0.082 & 0.021 \\
\hline$D^{l l}(3,3)$ & $\mathcal{I} \mathcal{G}$ & 0.020 & 2.000 & 1.864 & 2.230 & 3.136 & 4.541 & 5.612 & 2.601 & 3.260 \\
\hline$S^{\xi, \xi}(1,1)$ & $\mathcal{I G}$ & 0.002 & 0.200 & 0.171 & 0.183 & 0.203 & 0.227 & 0.242 & 0.203 & 0.204 \\
\hline$S^{\xi, \xi}(2,2)$ & $\mathcal{I G}$ & 0.002 & 0.200 & 0.080 & 0.084 & 0.091 & 0.099 & 0.105 & 0.090 & 0.091 \\
\hline
\end{tabular}

Notes: This table reports the priors and the posterior density for the parameters of $\Gamma S$ matrix in Eq. 1 The first three columns report the distributions, means and standard deviations for the prior distributions. The fourth to the eight columns report the .5-th, 5-th, the 50-th and the 95-th 99.5-th percentile of the posterior distributions, respectively. The last two columns report the modes and the means of the posterior distributions. All the statistics of all the posterior distribution are multiplied by 100. The results were obtained using the Metropolis Hastings algorithm. 
Table 11: Prior and Posterior Distribution of the Other Parameters

\begin{tabular}{|c|c|c|c|c|c|c|c|c|c|c|}
\hline & \multicolumn{3}{|c|}{ Prior } & \multicolumn{7}{|c|}{ Posterior } \\
\hline & Distr. & Mean & StdDev & $0.5 \%$ & $5 \%$ & $50 \%$ & $95 \%$ & $99.5 \%$ & Mode & Mean \\
\hline$\Lambda_{0}(1)$ & $\mathcal{N}$ & 0.000 & 20.000 & -6.23 & -5.31 & -2.49 & -0.06 & 1.54 & -3.03 & -2.67 \\
\hline$\Lambda_{0}(2)$ & $\mathcal{N}$ & 0.000 & 20.000 & -1.73 & 0.00 & 4.00 & 10.11 & 11.51 & 4.87 & 4.37 \\
\hline$\Lambda_{0}(3)$ & $\mathcal{N}$ & 0.000 & 20.000 & -1.45 & -1.09 & -0.28 & 0.65 & 1.18 & -0.44 & -0.24 \\
\hline$\Lambda_{0}(4)$ & $\mathcal{N}$ & 0.000 & 20.000 & -0.86 & -0.48 & 0.39 & 1.60 & 2.01 & 0.44 & 0.49 \\
\hline$\Lambda_{0}(5)$ & $\mathcal{N}$ & 0.000 & 20.000 & -0.61 & 0.22 & 1.01 & 2.23 & 3.80 & 1.19 & 1.00 \\
\hline$\Lambda_{0}(6)$ & $\mathcal{N}$ & 0.000 & 20.000 & -0.40 & -0.29 & 0.03 & 0.38 & 0.55 & -0.08 & 0.04 \\
\hline$\Lambda_{0}(7)$ & $\mathcal{N}$ & 0.000 & 20.000 & -1.43 & -0.52 & 0.84 & 2.00 & 3.61 & 0.83 & 0.77 \\
\hline$\Lambda_{0}(8)$ & $\mathcal{N}$ & 0.000 & 20.000 & -4.71 & -3.46 & -0.87 & 1.82 & 2.84 & -0.25 & -0.97 \\
\hline$\Lambda_{1}(1,6)$ & $\mathcal{N}$ & 0.000 & 50.000 & -30.69 & -13.82 & 24.84 & 59.67 & 80.53 & 35.32 & 27.89 \\
\hline$\Lambda_{1}(2,6)$ & $\mathcal{N}$ & 0.000 & 50.000 & -124.37 & -97.86 & -52.59 & 1.72 & 40.73 & -53.65 & -51.24 \\
\hline$\Lambda_{1}(3,6)$ & $\mathcal{N}$ & 0.000 & 50.000 & -1.10 & 10.93 & 32.82 & 51.62 & 58.34 & 30.85 & 33.01 \\
\hline$\Lambda_{1}(4,6)$ & $\mathcal{N}$ & 0.000 & 50.000 & -4.20 & 5.57 & 41.79 & 67.83 & 83.37 & 38.24 & 37.63 \\
\hline$\Lambda_{1}(5,6)$ & $\mathcal{N}$ & 0.000 & 50.000 & -39.15 & -31.50 & -16.60 & -3.17 & 4.93 & -14.15 & -16.80 \\
\hline$\Lambda_{1}(6,6)$ & $\mathcal{N}$ & 0.000 & 50.000 & 12.14 & 17.53 & 28.32 & 39.76 & 46.55 & 22.50 & 27.96 \\
\hline$\Lambda_{1}(7,6)$ & $\mathcal{N}$ & 0.000 & 50.000 & -77.00 & -65.81 & -41.71 & -9.16 & 5.28 & -35.51 & -40.36 \\
\hline$\Lambda_{1}(8,6)$ & $\mathcal{N}$ & 0.000 & 50.000 & -131.43 & -105.47 & -55.12 & -10.70 & 15.61 & -15.61 & -56.74 \\
\hline$\Sigma_{m}(4,4)$ & $\mathcal{U}^{*}$ & 0.000 & 0.005 & 0.001 & 0.001 & 0.004 & 0.011 & 0.015 & 0.001 & 0.005 \\
\hline$\Sigma_{m}(6,6)$ & $\mathcal{U}^{*}$ & 0.000 & 0.005 & 0.133 & 0.140 & 0.153 & 0.168 & 0.178 & 0.154 & 0.154 \\
\hline$\Sigma_{m}(7,7)$ & $\mathcal{U}^{*}$ & 0.000 & 0.005 & 0.187 & 0.196 & 0.216 & 0.238 & 0.251 & 0.211 & 0.216 \\
\hline$\Sigma_{m}(8,8)$ & $\mathcal{U}^{*}$ & 0.000 & 0.005 & 0.104 & 0.110 & 0.121 & 0.132 & 0.140 & 0.116 & 0.121 \\
\hline$\Sigma_{m}(9,9)$ & $\mathcal{U}^{*}$ & 0.000 & 0.005 & 0.001 & 0.002 & 0.010 & 0.027 & 0.038 & 0.004 & 0.012 \\
\hline$\Sigma_{m}(10,10)$ & $\mathcal{U}^{*}$ & 0.000 & 0.005 & 0.202 & 0.213 & 0.237 & 0.263 & 0.280 & 0.231 & 0.237 \\
\hline$\Sigma_{m}(11,11)$ & $\mathcal{U}^{*}$ & 0.000 & 0.005 & 0.354 & 0.383 & 0.440 & 0.488 & 0.498 & 0.447 & 0.438 \\
\hline$\Sigma_{m}(12,12)$ & $\mathcal{U}^{*}$ & 0.000 & 0.005 & 0.001 & 0.004 & 0.024 & 0.058 & 0.077 & 0.001 & 0.027 \\
\hline$\Sigma_{m}(13,13)$ & $\mathcal{U}^{*}$ & 0.000 & 0.002 & 0.001 & 0.004 & 0.024 & 0.064 & 0.091 & 0.008 & 0.028 \\
\hline$\Sigma_{m}(14,14)$ & $\mathcal{U}^{*}$ & 0.000 & 0.002 & 0.181 & 0.188 & 0.197 & 0.200 & 0.200 & 0.199 & 0.196 \\
\hline$X_{0}(4)$ & $\mathcal{U}^{*}$ & -0.015 & 0.015 & -0.012 & -0.006 & 0.008 & 0.014 & 0.015 & 0.014 & 0.007 \\
\hline$X_{0}(5)$ & $\mathcal{U}^{*}$ & -0.015 & 0.015 & -0.013 & -0.007 & 0.007 & 0.014 & 0.015 & 0.013 & 0.006 \\
\hline$X_{0}(6)$ & $\mathcal{U}^{*}$ & -0.100 & 0.200 & 0.025 & 0.065 & 0.137 & 0.189 & 0.198 & 0.127 & 0.133 \\
\hline$X_{0}(7)$ & $\mathcal{U}^{*}$ & -0.010 & 0.050 & -0.010 & -0.008 & -0.002 & 0.006 & 0.010 & -0.002 & -0.001 \\
\hline$X_{0}(8)$ & $\mathcal{U}^{*}$ & -0.010 & 0.050 & 0.028 & 0.032 & 0.037 & 0.044 & 0.047 & 0.037 & 0.037 \\
\hline $\bar{C}(4)$ & $\mathcal{U}^{*}$ & 0.000 & 0.015 & 0.005 & 0.006 & 0.008 & 0.010 & 0.012 & 0.008 & 0.008 \\
\hline $\bar{C}(5)$ & $\mathcal{U}^{*}$ & 0.000 & 0.015 & 0.002 & 0.002 & 0.004 & 0.005 & 0.006 & 0.004 & 0.004 \\
\hline $\bar{C}(6)$ & $\mathcal{U}^{*}$ & 0.000 & 0.120 & 0.008 & 0.036 & 0.080 & 0.098 & 0.110 & 0.100 & 0.075 \\
\hline$A(4)$ & $\mathcal{N}$ & 0.000 & 0.010 & -0.878 & -0.546 & 0.061 & 0.613 & 0.917 & 0.102 & 0.054 \\
\hline$A(14)$ & $\mathcal{N}$ & 0.000 & 0.002 & -0.121 & -0.101 & -0.067 & -0.033 & -0.015 & -0.055 & -0.067 \\
\hline
\end{tabular}

* : for the uniform distribution we report lower and upper bound of the support.

Notes: This table reports the priors and the posterior density for the parameters of $\Sigma_{m}$ in eq $15 \Lambda_{0}$ and $\Lambda_{1}$ in eq. 4 $C$ in eq. 1 and the initial values of the latent variables, $X_{0}$. The first three columns report the distributions, means and standard deviations of the prior distributions. The fourth to the eight columns report the .5-th, 5-th, the 50-th and the 95-th 99.5-th percentile of the posterior distributions, respectively. The last two columns report the modes and the means of the posterior distributions. The statistics of all the posterior distribution of $\Sigma_{m}$ and $A$ are multiplied by 100. For the uniform distribution the lower and upper bounds are reported instead of mean and standard deviation, respectively. The results were obtained using the Metropolis Hastings algorithm. 


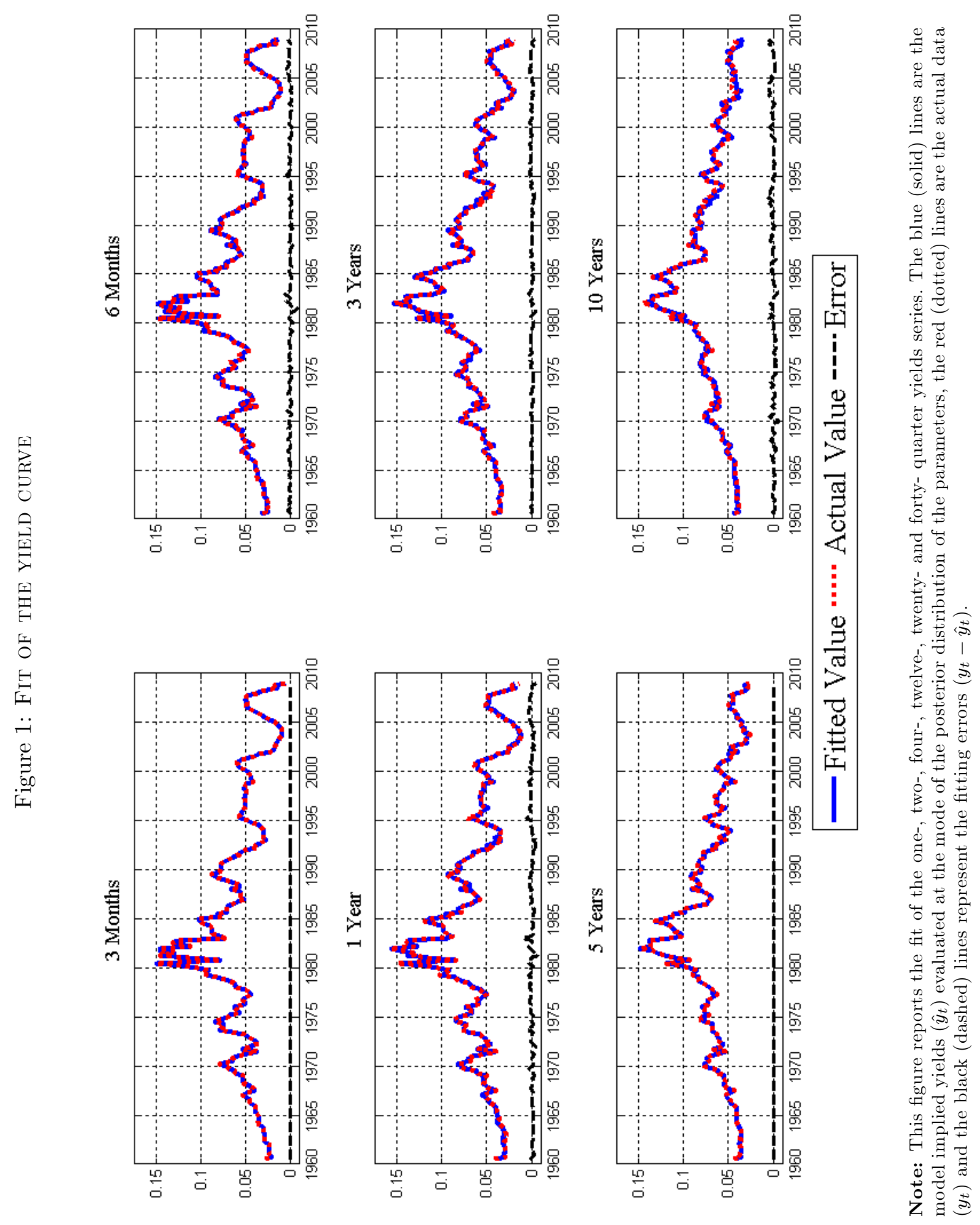




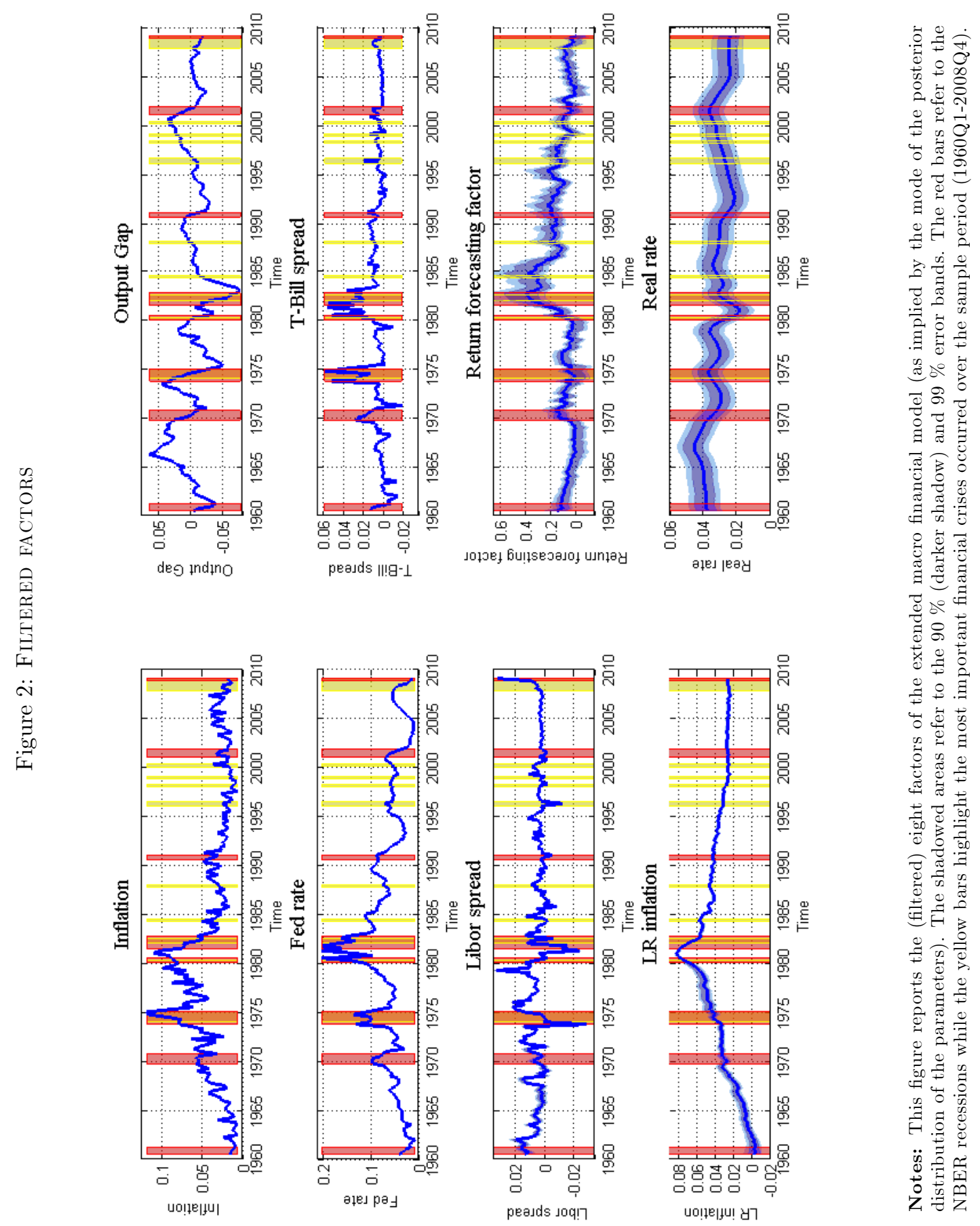




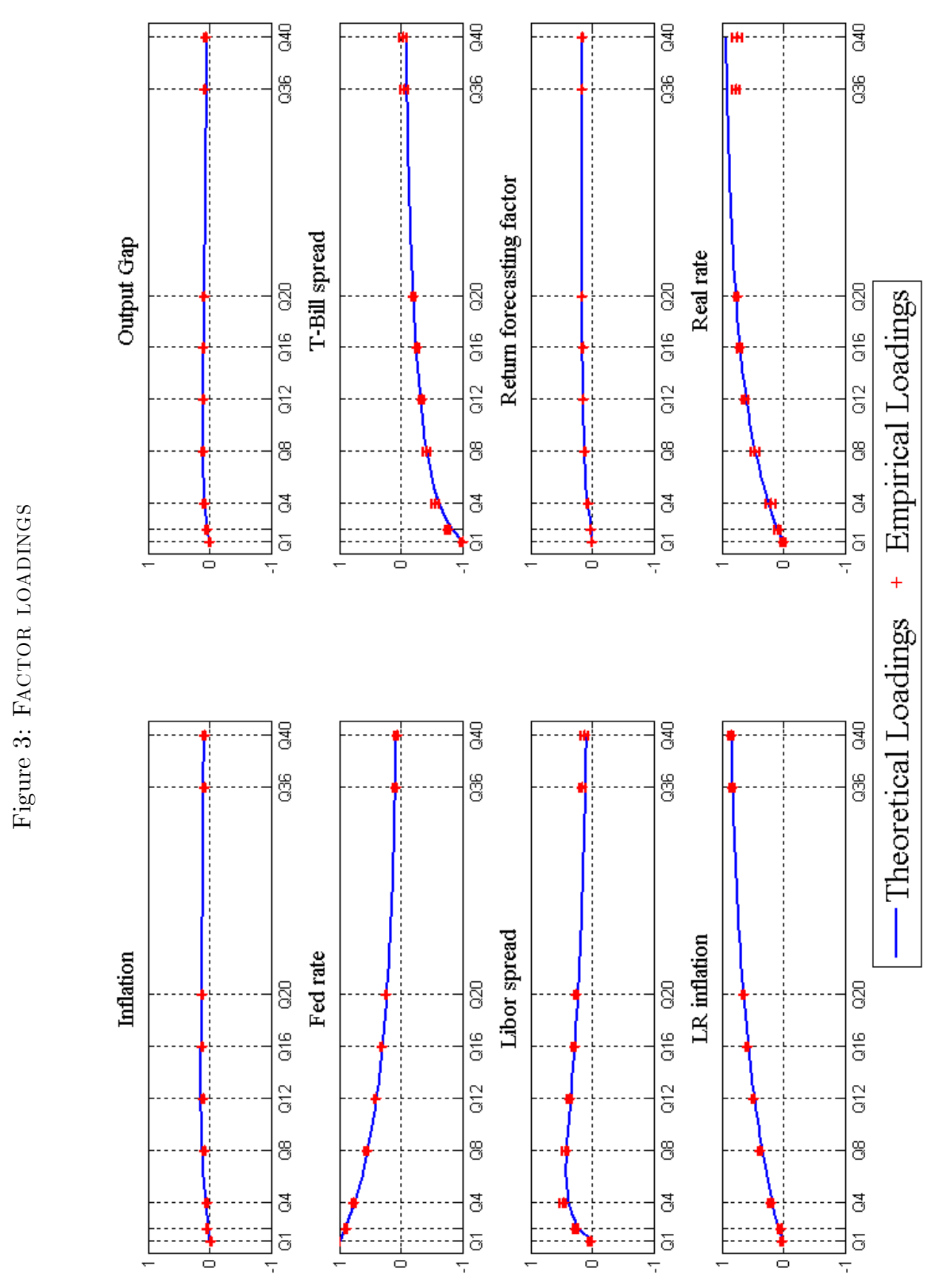

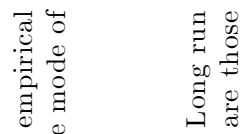

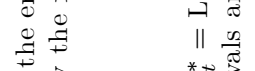

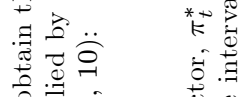
०.

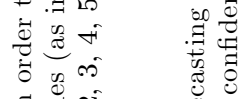

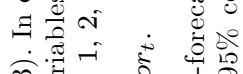

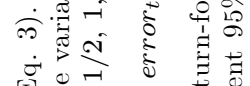

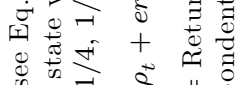

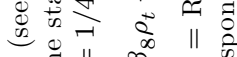

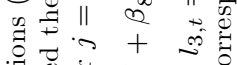

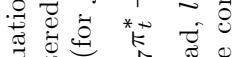

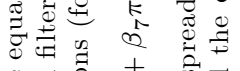

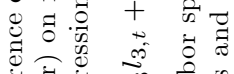

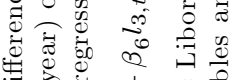
7 i  苛范告 次完 离

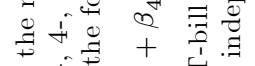
Bิ

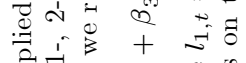

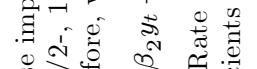
更

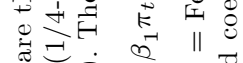
की 焉芯

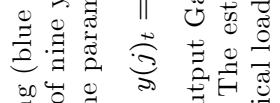
on

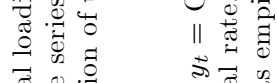

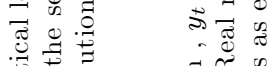
至

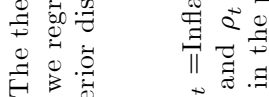

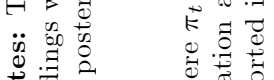

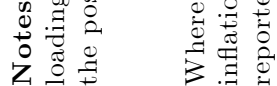




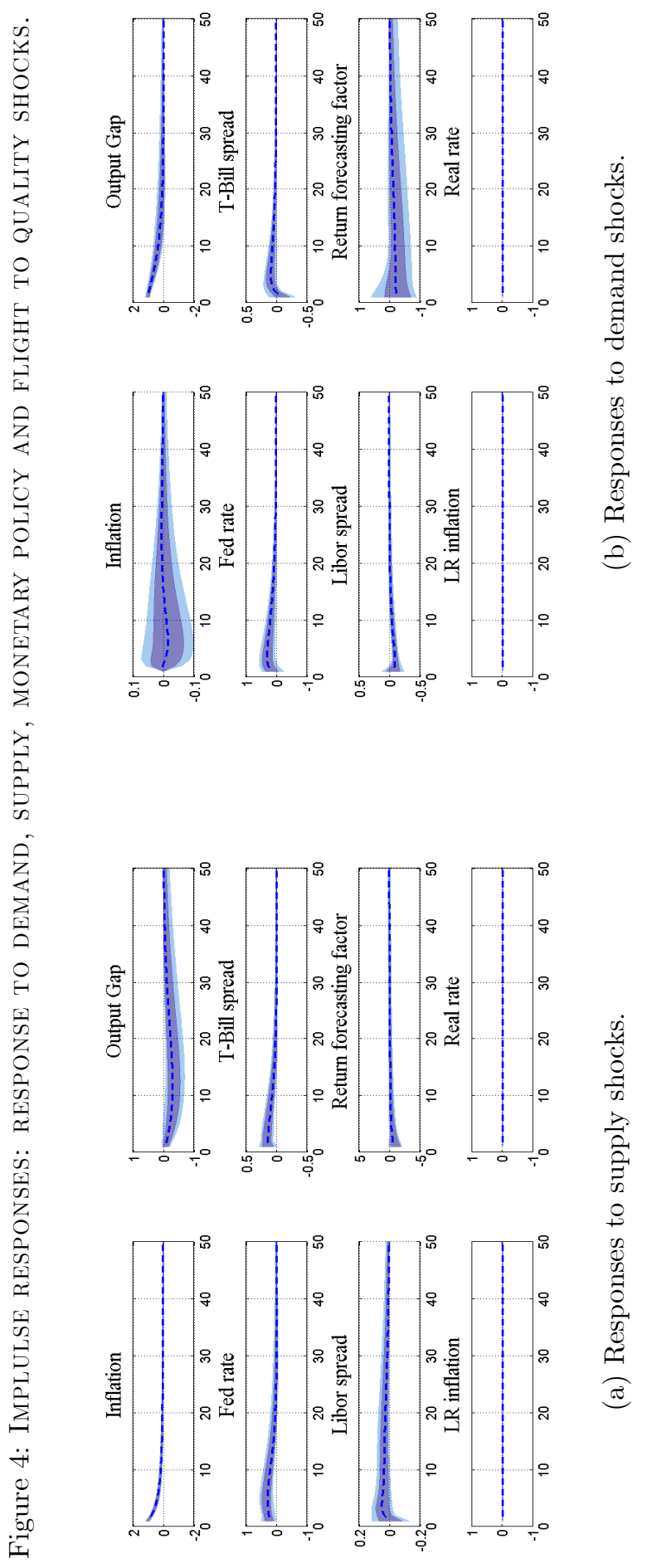

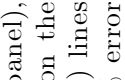

․ㅝ웡

$\because 8$

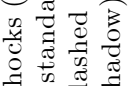

क क त $\frac{\pi}{0}$

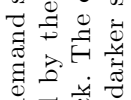

덩

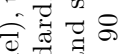

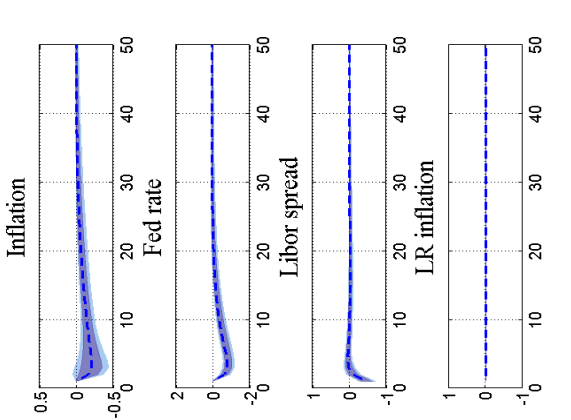

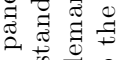

世

$\frac{1}{1} \approx \frac{g}{4}$

红岁悹

承光

क

定它记

क

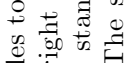

.

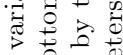

월

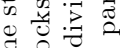

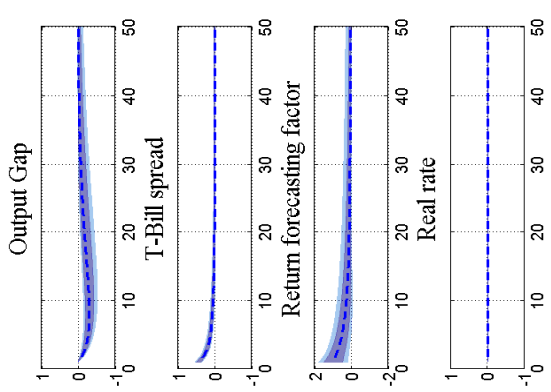

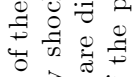

की

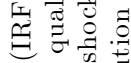

구워 군

茟苟

05

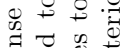

范范

馬

己.

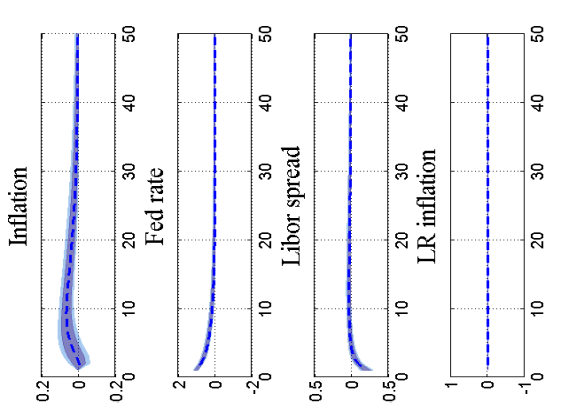

岁志政

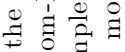

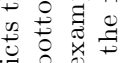

o 0

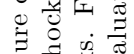

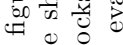

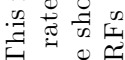

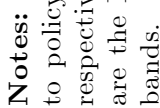




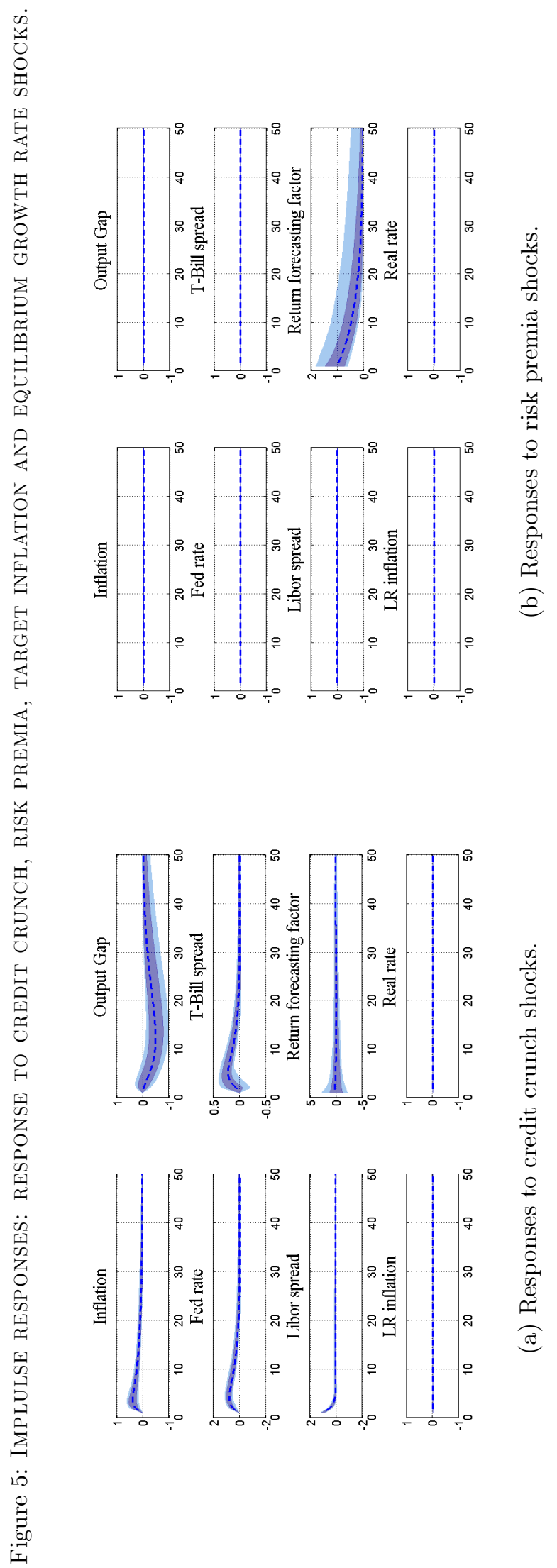

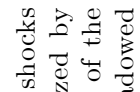

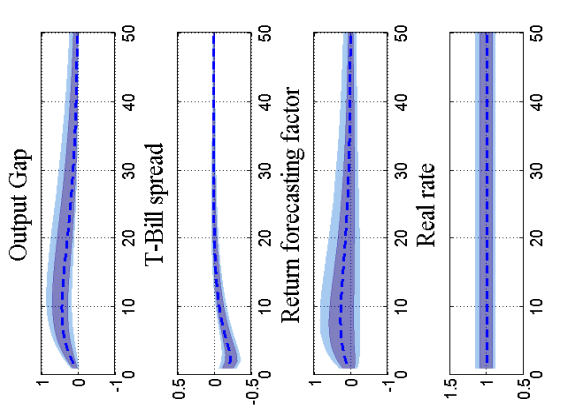

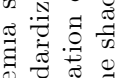

$\frac{\dot{m}}{\stackrel{9}{0}}$

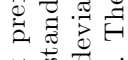

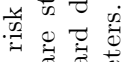

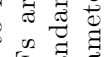

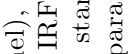

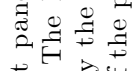

苟是它

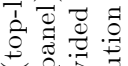

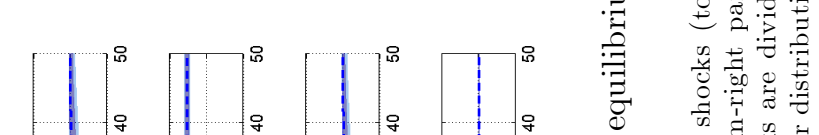

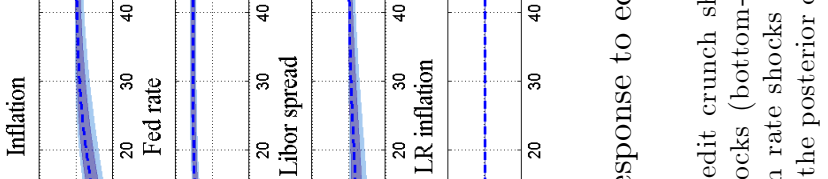

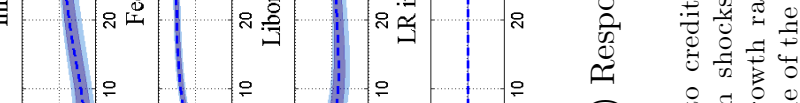

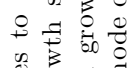

䙳

娄

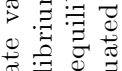

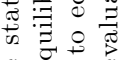

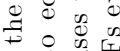

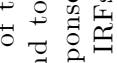

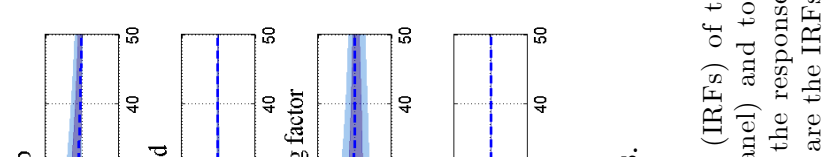

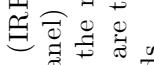

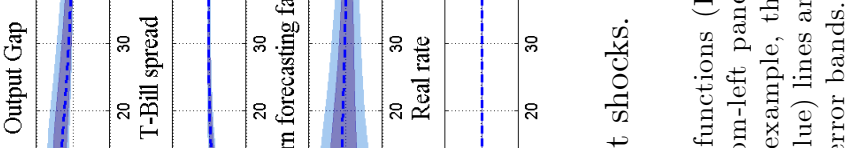

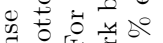

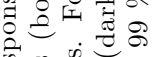

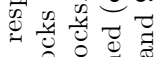

का

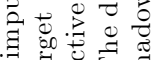

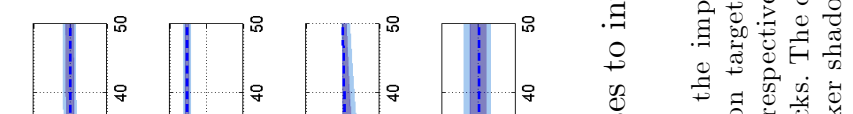

कि.

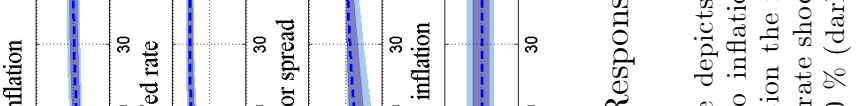

0.

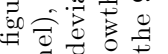

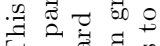

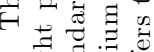

के

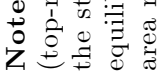



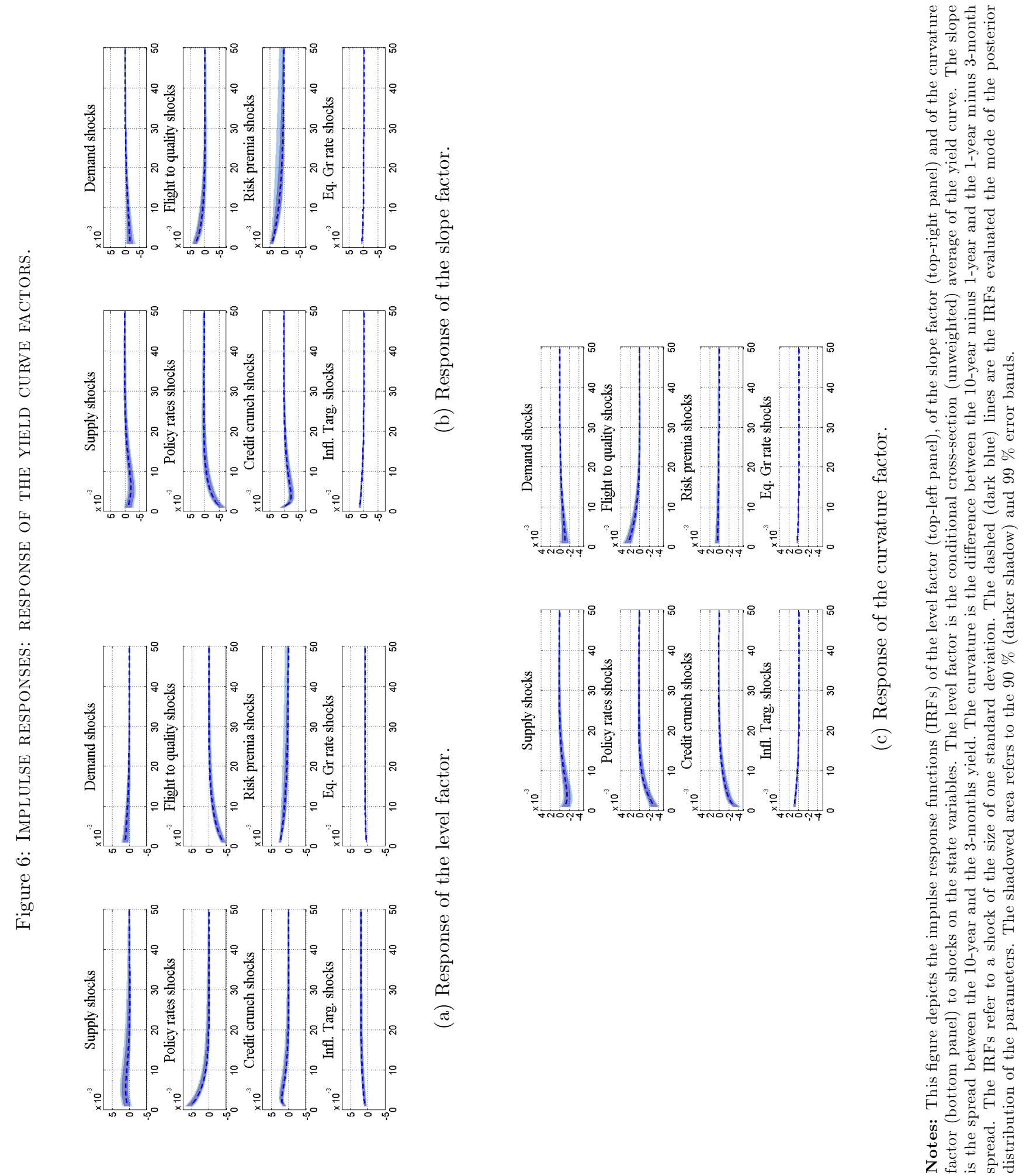
Figure 7: VARIANCE Decompositions

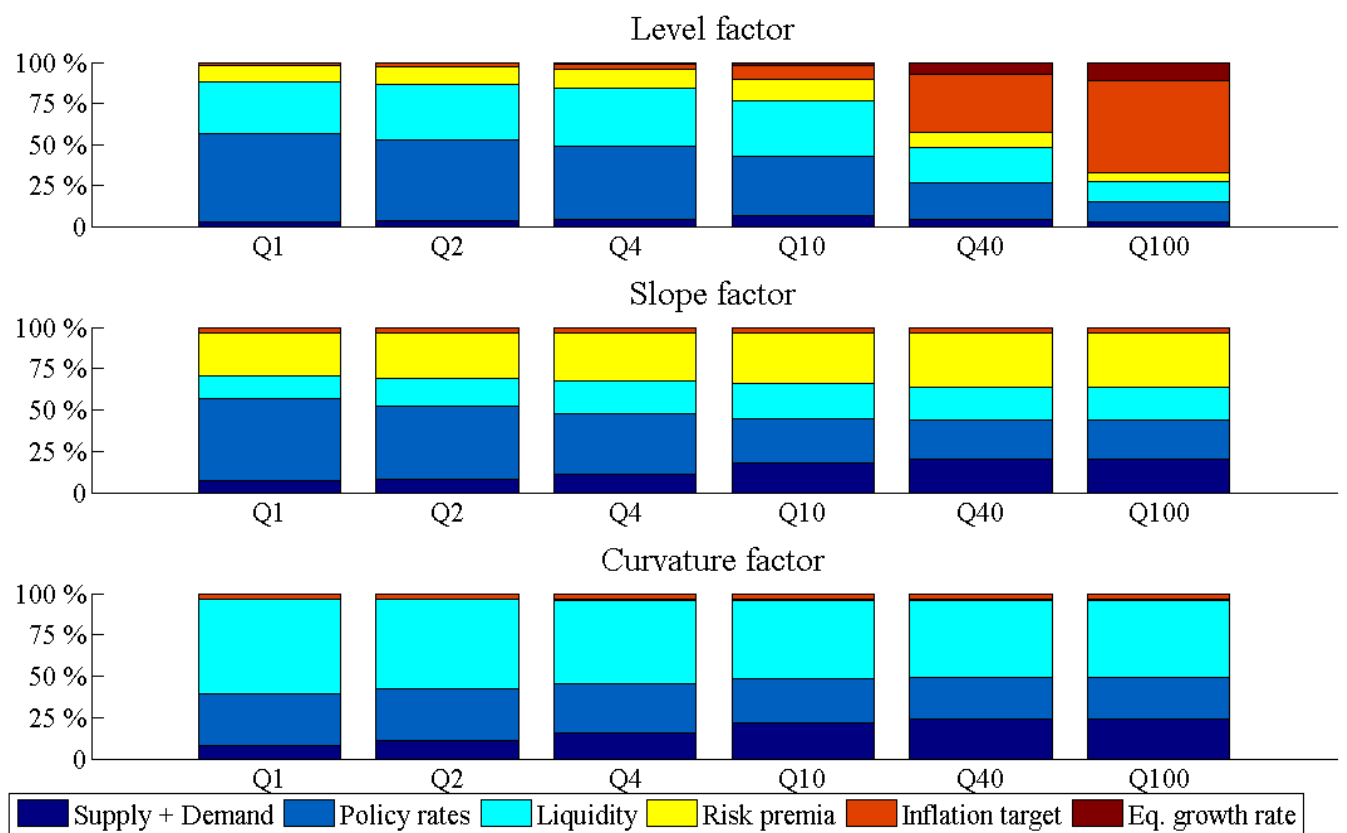

Notes: This figure displays the variance decomposition of the forecasting error of the level factor (top panel), of the slope factor (center panel) and of the curvature factor (bottom panel) evaluated the mode of the posterior distribution of the parameters. The level factor is the conditional cross-section (unweighted) average of the yield curve. The slope is the spread between the 10-year and the 3-months yield. The curvature is the difference between the 10-year minus 1-year and the 1-year minus 3-month spread. "Supply + Demand" stands for supply shocks plus demand shocks, "Policy rates" refers to policy rate shocks, "Liquidity" stands for flight to quality plus credit crunch shocks,"Inflation target" stands for inflation target shocks and "Eq. growth rate" refers to equilibrium growth rate shocks. 


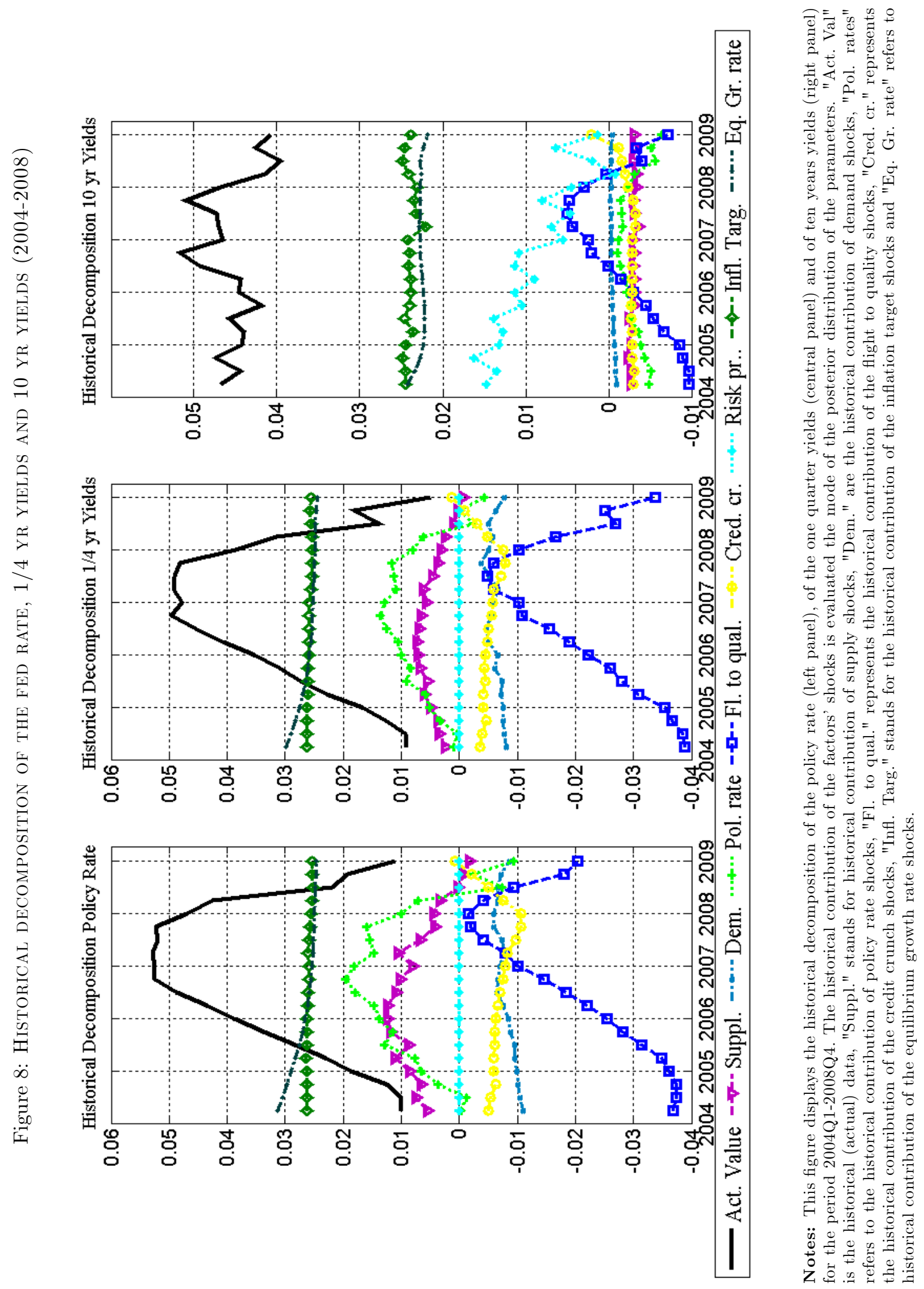


Figure 9: Variance Decompositions (sub-Sample)

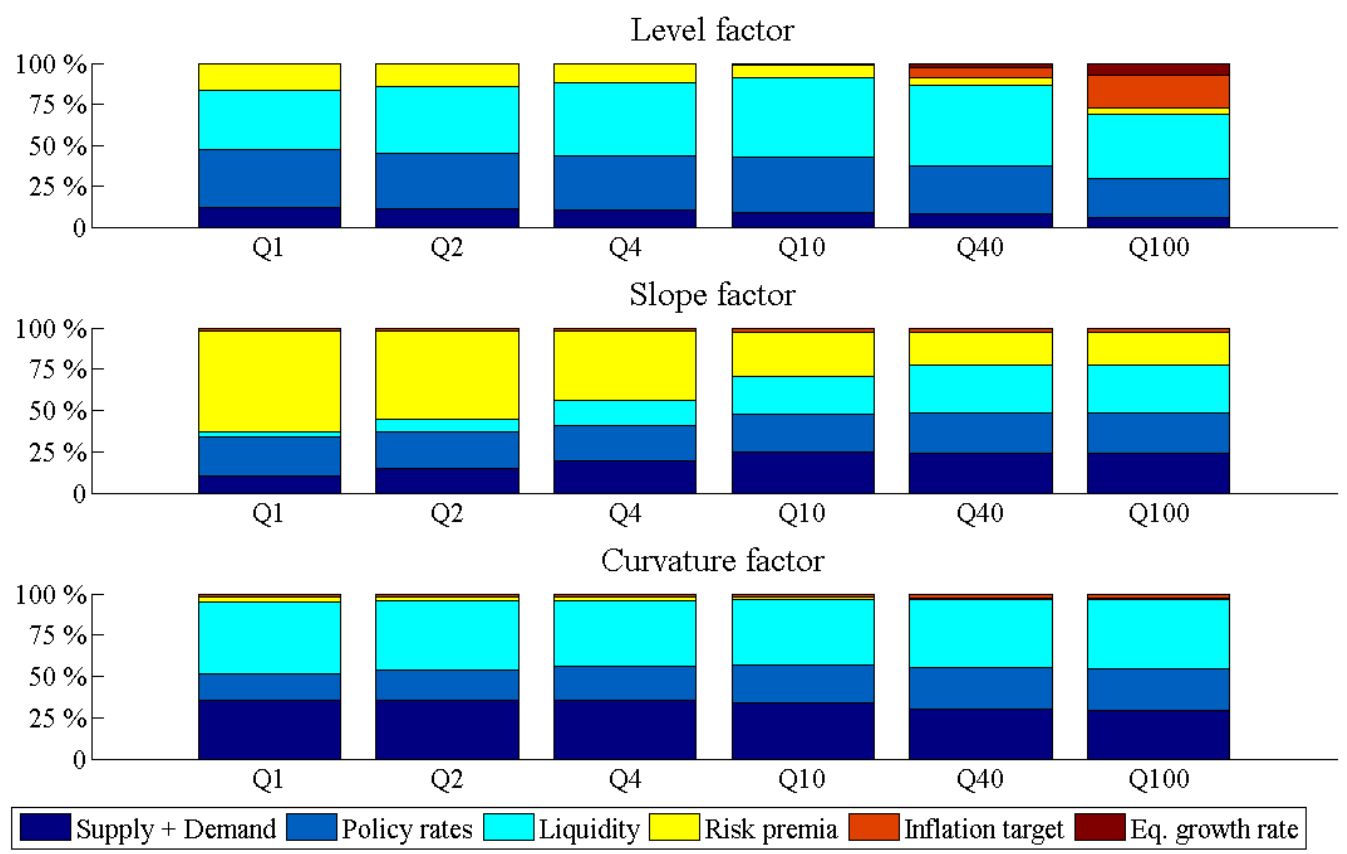

Notes: This figure displays the variance decomposition of the forecasting error of the level factor (top panel), of the slope factor (center panel) and of the curvature factor (bottom panel) for the subsample period 1986Q1-2008Q4. The variance decomposition is evaluated the mode of the posterior distribution of the parameters. The level factor is the conditional cross-section (unweighted) average of the yield curve. The slope is the spread between the 10-year and the 3 -months yield. The curvature is the difference between the 10-year minus 1-year and the 1-year minus 3-month spread. "Supply + Demand" stands for supply shocks plus demand shocks, "Policy rates" refers to policy rate shocks, "Liquidity" stands for flight to quality plus credit crunch shocks, "Inflation target" stands for inflation target shocks and "Eq. growth rate" refers to equilibrium growth rate shocks. 

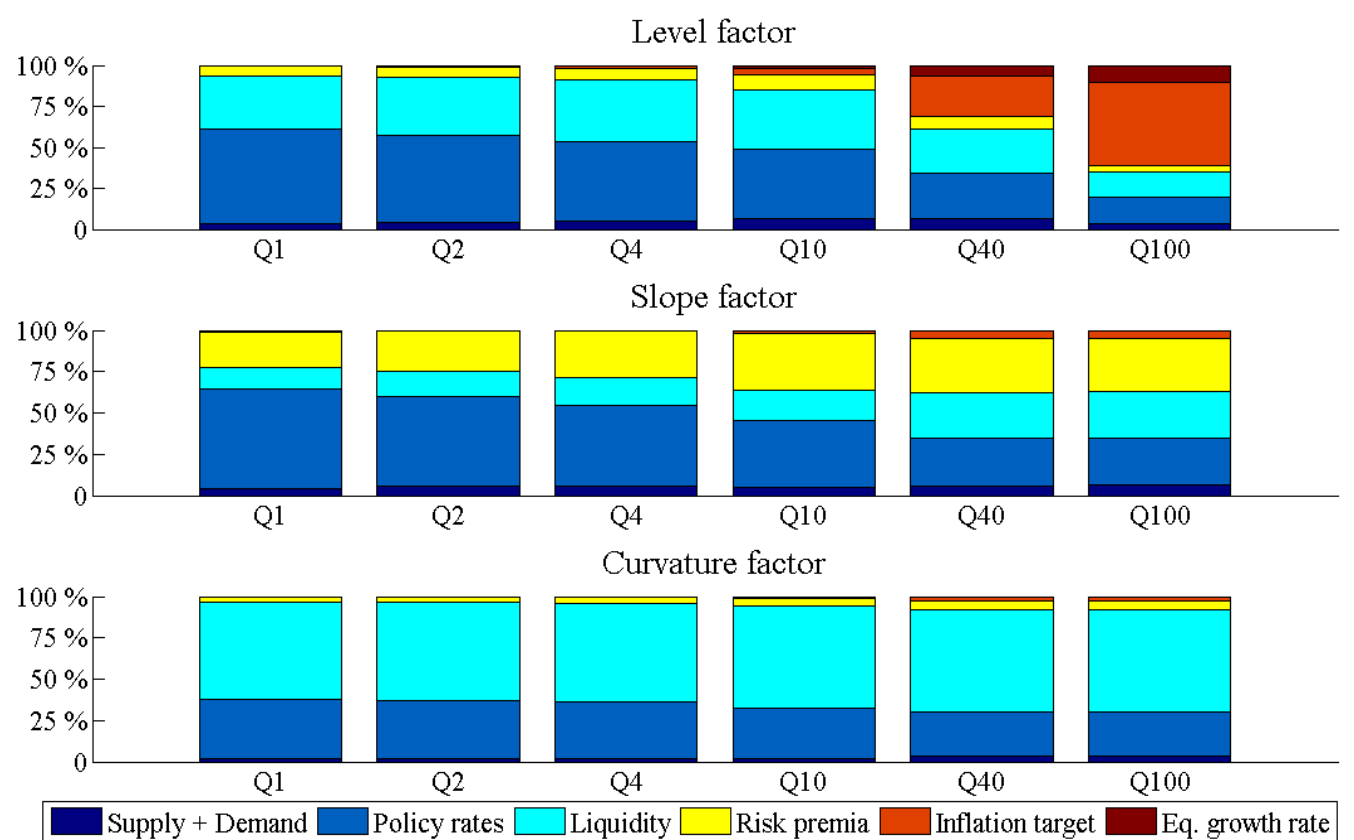

Notes: This figure displays the variance decomposition of the forecasting error of the level factor (top panel), of the slope factor (center panel) and of the curvature factor (bottom panel) for the sample period 1960Q1-2008Q4. The variance decomposition is evaluated the maximum of the likelihood function. The level factor is the conditional cross-section (unweighted) average of the yield curve. The slope is the spread between the 10-year and the 3-months yield. The curvature is the difference between the 10-year minus 1-year and the 1-year minus 3-month spread. "Supply + Demand" stands for supply shocks plus demand shocks, "Policy rates" refers to policy rate shocks, "Liquidity" stands for flight to quality plus credit crunch shocks, "Inflation target" stands for inflation target shocks and "Eq. growth rate" refers to equilibrium growth rate shocks. 